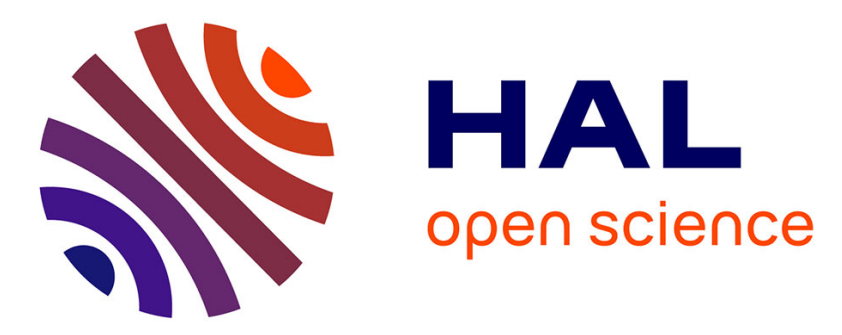

\title{
Coupling rigid bodies motion with single phase and two-phase compressible flows on unstructured meshes
}

Quentin Carmouze, François Fraysse, Richard Saurel, Boniface Nkonga

\section{To cite this version:}

Quentin Carmouze, François Fraysse, Richard Saurel, Boniface Nkonga. Coupling rigid bodies motion with single phase and two-phase compressible flows on unstructured meshes. Journal of Computational Physics, 2018, 375, pp.1314-1338. 10.1016/j.jcp.2018.08.023 . hal-02082701

\section{HAL Id: hal-02082701 https://hal.science/hal-02082701}

Submitted on 28 Mar 2019

HAL is a multi-disciplinary open access archive for the deposit and dissemination of scientific research documents, whether they are published or not. The documents may come from teaching and research institutions in France or abroad, or from public or private research centers.
L'archive ouverte pluridisciplinaire HAL, est destinée au dépôt et à la diffusion de documents scientifiques de niveau recherche, publiés ou non, émanant des établissements d'enseignement et de recherche français ou étrangers, des laboratoires publics ou privés. 


\title{
Coupling rigid bodies motion with single phase and two-phase compressible flows on unstructured meshes
}

\author{
Quentin Carmouze ${ }^{(1,2,3)}$, François Fraysse ${ }^{(2)}$, Richard Saurel ${ }^{(1,2)}$ and Boniface Nkonga ${ }^{(3)}$ \\ (1) Aix Marseille Univ, CNRS, Centrale Marseille, LMA, Marseille, France \\ ${ }^{(2)} \mathrm{RS} 2 \mathrm{~N}$, Chemin de Gaumin, Saint-Zacharie 83640, France \\ ${ }^{(3)}$ University of Nice, LJAD UMR CNRS 7351, Parc Valrose, 06108 Nice Cedex, France
}

\begin{abstract}
A simple method is developed to couple accurately the motion of rigid bodies to compressible fluid flows. Solid rigid bodies are tracked through a Level-Set function. Numerical diffusion is controlled thanks to a compressive limiter (Overbee) in the frame of MUSCL type scheme, giving an excellent compromise between accuracy and efficiency on unstructured meshes (Chiapolino et al., 2017). The method requires low resolution to preserve solid bodies' volume. Several coupling methods are then addressed to couple rigid body motion to fluid flow dynamics: a method based on stiff relaxation and two methods based on Ghost cells (Fedkiw et al., 1999) and immersed boundaries. Their accuracy and convergence rates are compared against an immersed piston problem in 1D having exact solution. The second Ghost cell method is shown to be the most efficient. It is then extended to multidimensional computations on unstructured meshes and its accuracy is checked against flow computations around blunt bodies. Reference results are obtained when the flow evolves around a rigid body at rest. The same rigid body is then considered with prescribed velocity moving in a flow at rest. Computed results involving wave dynamics match very well. The method is then extended to two-way coupling and illustrated to several examples involving shock wave interaction with solid particles as well as phase transition induced by projectiles motion in liquid-gas mixtures.
\end{abstract}

e-mails:

Quentin.carmouze@rs2n.eu

Francois.fraysse@rs2n.eu

Richard.saurel@univ-amu.fr

Boniface.nkonga@unice.fr 


\section{1 - Introduction}

In fluid mechanics two approaches are used to address the relative motion between a rigid body and a fluid. The first one is also the most commonly used and consists in considering a fluid moving around a body at rest. Setting appropriate boundary conditions at inflows, outflows and walls this method gives reliable results. A fundamental difficulty emerges rapidly as soon as two (or more) rigid bodies are present. For instance, a moving body in the presence of a distant wall at rest is problematic.

In the present approach rigid bodies are tracked on a fixed mesh with the help of Level-Set-type functions (Osher and Fedkiw, 2001). This function enables detection of fluids, solids and mixed cells. There are several advantages:

- The method allows solid body motion on fixed meshes and thus eliminates issues related to Lagrangian and ALE methods (Baum et al., 1994, Nkonga and Guillard, 1994, Nkonga, 2000);

- Surfaces are defined implicitly rather than explicitly as in the frame of Front Tracking (Glimm et al., 1998) and Interface Reconstruction methods (Youngs, 1984).

There are obviously drawbacks such as:

- Numerical smearing of the interface contour, that may result in solid body disappearance if the Level-Set function is resolved with insufficient accuracy ;

- Interface roughness effects due to mixed cells.

These issues become pregnant when dealing with unstructured grids as it is more difficult to control artificial smearing and roughness.

Recently a compressive limiter was introduced to sharpen diffuse interfaces in compressible two-phase flow modelling in the frame of 'diffuse interfaces' (Chiapolino et al., 2017, Saurel and Pantano, 2018). This limiter showed enhanced capturing properties with 2-3 cells only in the interfacial zone, when used in the frame of MUSCL type schemes and unstructured meshes. It is thus considered in the present contribution to solve the Level-Set function to control numerical smearing. Its ability to preserve volume and maintain shapes is examined and will be shown to be reasonably accurate.

The coupling between solid body motion and compressible fluid flow is then examined. It is first examined in one-way, with prescribed solid velocity and action on the surrounding fluid. Three methods of coupling are examined:

- The first one is also the simplest and considers stiff velocity relaxation between the fluid and solid.

- The second one considers Ghost cells in the solid where specific fluid state is prescribed in a given band of cells closed to the interface.

- The third one consists in an improvement of the former to improve its convergence. The Ghost state is modified to improve the surface pressure computation, improving shock and rarefaction waves formation in the fluid during impulsive motion.

Comparison of the various coupling methods is done in 1D with the help of an exact solution of an immersed piston set to impulsive motion, quite similar to the exact shock tube solution.

The coupling method is then extended to multi-D, posing extra difficulties as sliding effects between solid and fluid have to be considered in a context where the interface is arbitrarily rough, as a consequence of unstructured mesh.

The coupling method when the solid is moving in a fluid at rest is validated by comparing computational results when the solid is at rest and the fluid is moving through appropriate boundary conditions, as done in most CFD computation. It is then extended to two-way coupling, through the computation of pressure force integral over the solid surface. It enables update of the solid body velocity which in turn affects the fluid flow. Computational examples of shock - solid particles interaction are shown to illustrate method's capability.

In the area of solid-fluid coupling with Level-Set type methods, many contributions have to be mentioned such as for example, Liu et al. (2003), Wang et al. (2006), Liu et al. (2006), Liu et al. (2008), Zeng and Farhat (2012) this list being certainly not exhaustive. However it seems that important differences appear 
with the present contribution. First, Cartesian grids are considered instead of unstructured ones. Second, exact or approximate local Riemann problem solution is set in mixture cells to enforce interface conditions. In the present contribution, such ingredient is not used, this detail being important when dealing with sophisticated flow models, such as multiphase flow ones. Last, Ghost Cells in multi-D computations are filled with fluid state normal to the interface in a band (or layer) of cells of finite size. Determination of these cells in the normal direction to the interface may be challenging when dealing with unstructured grids. In the present contribution this issue is replaced by a simple averaging method.

Fluid-fluid and solid-fluid coupling with Level-Set methods have been addressed in the frame of unstructured meshes by Farhat et al. (2008, 2012), Wang et al. (2011) and possibly other authors. It seems that similar restrictions as the former lists with Cartesian grid approaches are present:

- Use of local Riemann problem solution,

- Sophisticated method for setting fluid state in the Ghost-Cell band.

The present approach doesn't seem more accurate than existing ones but seems conceptually simpler and easier to implement.

The paper is organized as follows. The Level-Set method and its numerical resolution are summarized in Section 2. Then, coupling methods are examined in Section 3. The compressible flow model is presented in this section and a reference solution is built to address an immersed piston set to motion impulsively. Three different coupling methods are detailed and tested against the exact solution of the immersed piston test. The method that matches best the results is then extended to multi-dimensions in Section 4. This section ends by validations of the coupling method in $2 \mathrm{D}$ with a supersonic two-phase flow. Section 5 extends to coupling method to two-way coupling through pressure force computation over each rigid body surface. Conclusions are given in Section 6.

\section{2- Motion of rigid bodies}

Rigid bodies are tracked through the Level-Set function denoted by $\Phi$, that is in the present approach aimed to model a Heaviside function. Let us consider a domain $\Omega$ having a subdomain $\Omega_{\mathrm{f}}$ occupied by the fluid and another sub-domain $\Omega_{\mathrm{S}}$ occupied by the solid body, as schematized in Fig. 2.1.

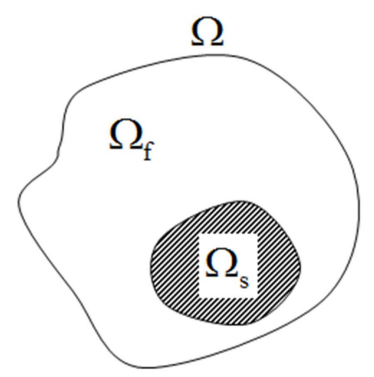

Figure 2.1 - Schematic representation of the solid and fluid sub-domains

The Level-Set function $\Phi$ indicates the presence of materials and interface at a given point of space $\mathbf{M}$. It is defined as,

$$
\begin{cases}1 \geq \Phi>0 & \text { if } M \in \Omega_{\mathrm{s}} \\ 0 & \text { if } M \in \Omega_{\mathrm{f}} \cap \Omega_{\mathrm{s}} \\ 0>\Phi \geq-1 & \text { if } M \in \Omega_{\mathrm{f}}\end{cases}
$$

With these definitions the zero level pays particular attention as it represents the solid-fluid interface. The Level-Set function obeys the transport equation,

$$
\frac{\partial \Phi}{\partial \mathrm{t}}+\overrightarrow{\mathrm{u}_{\mathrm{s}}} \cdot \vec{\nabla} \Phi=0
$$


where $\overrightarrow{\mathrm{u}_{\mathrm{s}}}$ denotes the solid body velocity. As it is constant in the rigid body and only time dependent, Eq. (2.2) can be expressed in conservation form:

$$
\frac{\partial \Phi}{\partial \mathrm{t}}+\vec{\nabla} \cdot\left(\Phi \overrightarrow{\mathrm{u}_{\mathrm{s}}}\right)=0
$$

The main difficulty with the Level-Set method is to preserve the level 0 . As a Heaviside function is initially set as $\Phi=1$ in the solid and $\Phi=-1$ in the fluid, numerical smearing of the discontinuity may result rapidly in solid volume loss. As soon as two interfaces are present solid body may disappear as time evolves. Several methods are available to balance this weakness:

- When the Level-Set function is used as a distance function (different of definition in Eq. (2.1)), a re-initialization procedure is able to restore the correct function profile (Osher and Fedkiw, 2001).

- When it is aimed to model a Heaviside function, as in the present work, the interface can be sharpened with the help of artificial compressibility terms (Olsson et al., 2007, Shukla et al., 2010). However these procedures require efforts, in particular in unstructured meshes and are consuming in computer resources.

For the sake of simplicity we adopt the method developed in Chiapolino et al. (2017) in the frame of diffuse interface modelling. This method was precisely designed to lower the numerical diffusion of so called 'diffuse interfaces' through a specific limiter, used to sharpen volume fraction profiles. This limiter (Overbee) is used in MUSCL type schemes (Van Leer, 1979) that are quite simple to implement in unstructured codes. Details of the implementation used in the present work are given in Chiapolino et al. (2017). The Overbee limiter is illustrated in Fig. 2.2 and corresponds to the upper bound of the first-order TVD region.

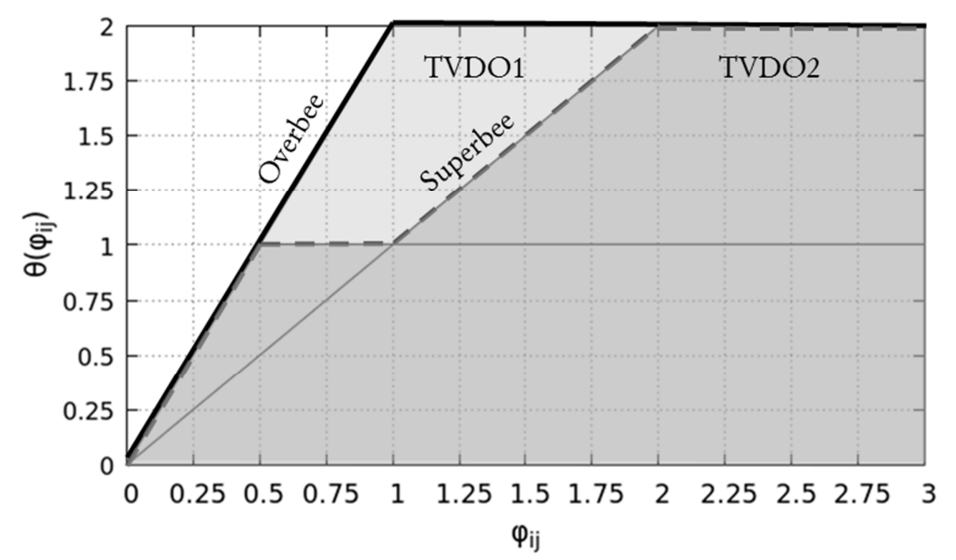

Figure 2.2- First-order and second-order TVD regions. The upper bounds of these regions correspond respectively to the Overbee and Superbee limiters.

The Overbee limiter used in the computational examples of the present paper reads:

$\theta\left(\varphi_{\mathrm{ij}}\right)=\max \left[0, \min \left[2 \varphi_{\mathrm{ij}}, 2\right]\right]$,

where $\varphi_{\mathrm{ij}}$ represents the ratio of slopes between cells $i$ and $j$.

Efficiency of this limiter is illustrated in Fig. 2.3 where a comparison with Superbee is shown for the transport of a Heaviside function at prescribed velocity. Superbee was considered as the optimum bound for the design of limiters (Sweby, 1984). However, when dealing with Heaviside functions only this upper bound can be overpassed, resulting in significant improvements of the solution, free of robustness issues. 

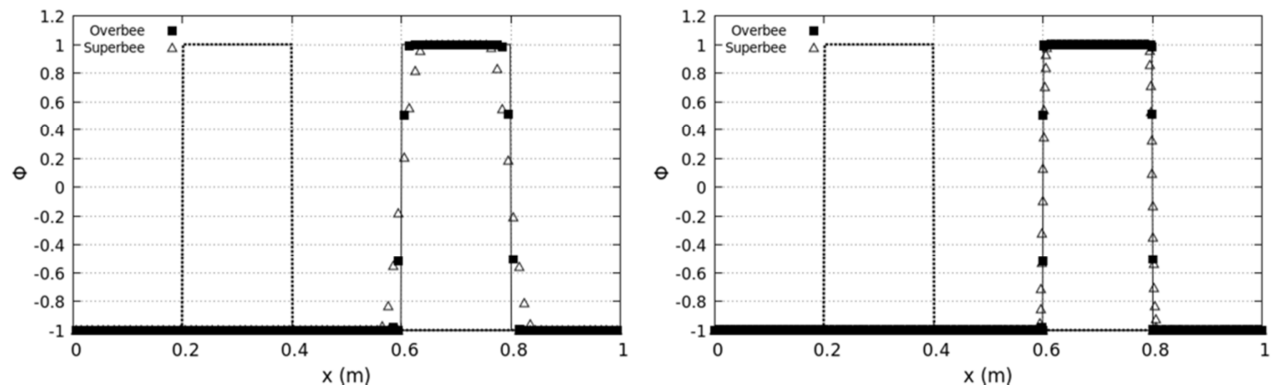

Figure 2.3- Comparison of the Overbee and Superbee limiters for the transport of a Heaviside function, here a Level-Set-type function. The advection speed is $100 \mathrm{~m} / \mathrm{s}$. The dashed lines represent the initial condition. The full line represents the exact solution. Gradients of the Heaviside function are computed with the least squares method, corresponding in the one-dimensional case to central approximations. Final time: $\mathrm{t}=4 \mathrm{~ms}, \mathrm{CFL}=0.8$. On the graph at left 100 cells are used while on the one at right

1000 cells are used. Overbee captures the discontinuity with two points only whatever the mesh resolution is.

In these computations, the gradients are computed with central approximations. Indeed, central differences correspond to the least square approximation method that preserves accuracy and robustness in unstructured meshes codes (Barth and Jespersen, 1989). It appears that the Overbee limiter handles discontinuities in two points only for any mesh refinement and any method of gradient computation (central differencing as well as upwind-downwind). Its capabilities in multi-D are excellent as well, as shown in Fig. 2.4 where a Zalesak (1979) disc is transported at prescribed velocity $(10 \mathrm{~m} / \mathrm{s})$ on an unstructured grid made of 16156 triangles.
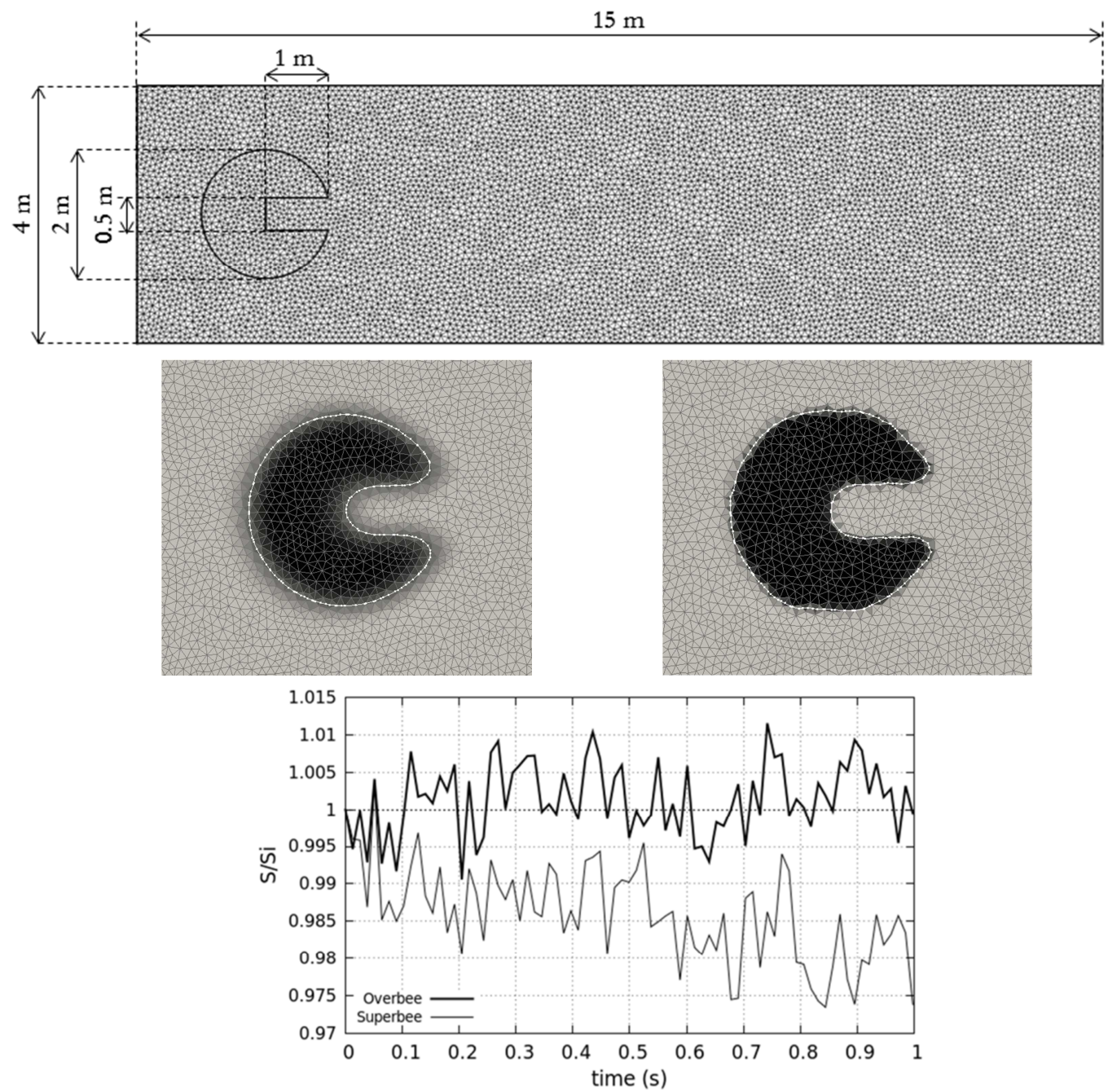

Figure 2.4- Transport of the Zalesak disc on an unstructured grid. The initial data and dimensions are shown on the upper graph. Contours of the Level-Set function and its zero level are shown in the graphs at the middle, at time 1s, before exiting the right boundary of the domain. The graph at left is obtained with the MUSCL-Superbee scheme while the one at right uses Overbee. 
The graph at bottom shows volume preservation. Superbee results in volume loss while Overbee preserves volume in the time average sense. All computations use least-square methods for the gradients computation, with an extended set of neighbors as defined in Fig. 2.5. The same mesh with 16156 cells is used in both computations, corresponding to an average cell size of 0.05

$\mathrm{m}$. The time step is computed to fulfil CFL restriction of 0.9 .

It also appears that the overall shape is well preserved. It is worth to mention that the two stencils described in Fig. 2.5 have been considered for the gradients computation in the least square method. The results are very similar, the final shape being slightly smoother with extended neighbors. Gradients computed with extended neighbors are always more accurate, having negligible extra cost in 2D computations and about 10\% extra cost in 3D. These two stencils are illustrated in the Fig. 2.5.

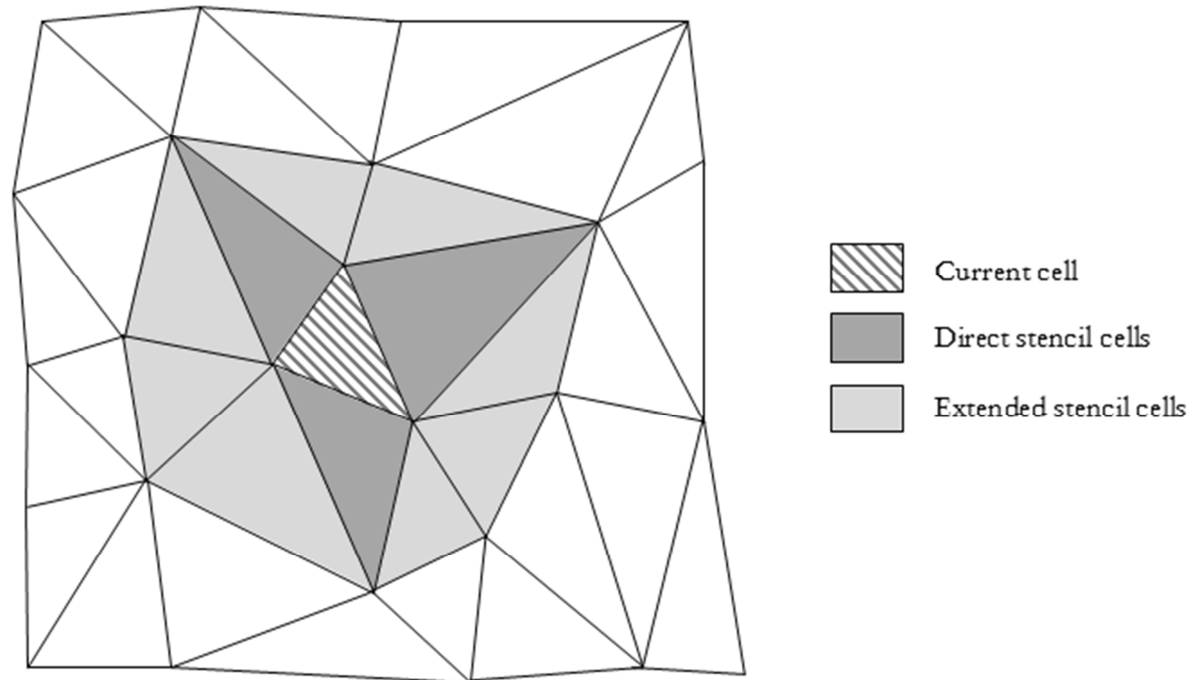

Figure 2.5- Definition of the two stencils: direct and extended neighbors.

Having now in hands a simple and efficient method to track rigid bodies, we now address coupling with the flow dynamics.

\section{3 - Coupling methods}

Solid-fluid coupling methods are now examined in the frame of a flow model that includes Euler and reactive Euler equations as well as multiphase mixtures in mechanical and thermal equilibrium. This formulation is particularly interesting to address phase transition at interfaces and in finely dispersed mixtures (Le Martelot et al., 2014, Saurel et al., 2016). In the present analysis, phase transition is omitted and coupling methods are analyzed in $1 \mathrm{D}$.

\subsection{Flow model}

The flow model, augmented by Level-Set equation reads,

$$
\begin{aligned}
& \frac{\partial \rho}{\partial \mathrm{t}}+\vec{\nabla} \cdot(\rho \overrightarrow{\mathrm{u}})=0 \\
& \frac{\partial \rho \overrightarrow{\mathrm{u}}}{\partial \mathrm{t}}+\vec{\nabla} \cdot(\rho \overrightarrow{\mathrm{u}} \otimes \overrightarrow{\mathrm{u}}+\mathrm{p} \overline{\mathrm{I}})=\overrightarrow{0} \\
& \frac{\partial \rho \mathrm{E}}{\partial \mathrm{t}}+\vec{\nabla} \cdot((\rho \mathrm{E}+\mathrm{p}) \overrightarrow{\mathrm{u}})=0 \\
& \frac{\partial \rho \mathrm{Y}_{\mathrm{k}}}{\partial \mathrm{t}}+\vec{\nabla} \cdot\left(\rho \mathrm{Y}_{\mathrm{k}} \overrightarrow{\mathrm{u}}\right)=0 \\
& \frac{\partial \Phi}{\partial \mathrm{t}}+\vec{\nabla} \cdot\left(\Phi \overrightarrow{\mathrm{u}}_{\mathrm{s}}\right)=0
\end{aligned}
$$


In these notations index $\mathrm{k}$ represents a given fluid constituent (liquid or gas). $\rho$ denotes the mixture density, $\vec{u}$ and $\vec{u}_{s}$ represent the velocity vector of the fluid and the solid respectively, $Y_{k}$ represent the mass fraction constituent $k$ and $E$ the total energy of the fluid mixture $\left(E=\sum_{k} Y_{k} e_{k}+\frac{1}{2} \vec{u} . \vec{u}\right)$.

Each fluid is assumed to be governed by a convex equation of state (EOS). Here the stiffened-gas EOS is retained for each constituent as it represents reasonably the thermodynamics of liquids in limited ranges of temperature (typically 300-500K). It also includes the ideal gas EOS when some parameters are set to zero. For a given constituent it reads,

$$
\mathrm{p}_{\mathrm{k}}=\left(\gamma_{\mathrm{k}}-1\right) \rho_{\mathrm{k}}\left(\mathrm{e}_{\mathrm{k}}-\mathrm{q}_{\mathrm{k}}\right)-\gamma_{\mathrm{k}} \mathrm{p}_{\mathrm{k}, \infty},
$$

where $\gamma_{\mathrm{k}}, \mathrm{q}_{\mathrm{k}}$ and $\mathrm{p}_{\mathrm{k}, \infty}$ are characteristic of a given constituent. A method to determine these parameters for liquid-vapor systems is given in Le Metayer et al. (2003). The stiffened-gas EOS can be improved to account for short distance repulsive effects, while remaining convex (Le Metayer and Saurel, 2016).

Under the assumption of temperature and pressure equilibrium among the phases, the following mixture EOS is obtained from the definition of mixture internal energy $\left(e=\sum_{k} Y_{k} e_{k}(T, p)\right)$ and mixture specific volume $\left(\mathrm{v}=\sum_{\mathrm{k}} \mathrm{Y}_{\mathrm{k}} \mathrm{v}_{\mathrm{k}}(\mathrm{T}, \mathrm{p})\right)$ definitions (Saurel et al., 2016):

$p\left(\rho, e, Y_{k}\right)=\frac{\sigma+\sqrt{\sigma^{2}+4 \overline{C_{v}} \omega}}{2 \overline{C_{v}}}$ with $\left\{\begin{array}{c}\sigma=\frac{e-\bar{q}}{v}\left(\overline{C_{p}}-\overline{C_{v}}\right)-p_{1, \infty} \overline{C_{v}}-p_{1, \infty} Y_{1}\left(C_{p, 1}-C_{v, 1}\right) \\ \omega=\frac{e-\bar{q}}{v} p_{1, \infty}\left(\overline{C_{p}}-\overline{C_{v}}-Y_{1}\left(C_{p, 1}-C_{v, 1}\right)\right)\end{array}\right.$

and $\overline{\mathrm{C}_{\mathrm{v}}}=\sum_{\mathrm{k}=1}^{\mathrm{N}} \mathrm{Y}_{\mathrm{k}} \mathrm{C}_{\mathrm{v}, \mathrm{k}}, \overline{\mathrm{C}_{\mathrm{p}}}=\sum_{\mathrm{k}=1}^{\mathrm{N}} \mathrm{Y}_{\mathrm{k}} \mathrm{C}_{\mathrm{p}, \mathrm{k}}, \overline{\mathrm{q}}=\sum_{\mathrm{k}=1}^{\mathrm{N}} \mathrm{Y}_{\mathrm{k}} \mathrm{C}_{\mathrm{v}, \mathrm{k}}$

The mixture temperature $\mathrm{T}=\mathrm{T}(\rho, \mathrm{p}, \mathrm{Y})$ is obtained as,

$$
T\left(\rho, p, Y_{k}\right)=\frac{1}{\rho\left(\sum_{k=1}^{N} \frac{Y_{k}\left(\gamma_{k}-1\right) C_{v, k}}{p+p_{k, \infty}}\right)}
$$

This EOS is valid when the liquid phase is denoted by index 1, the other constituents being ideal gases ( $\left.\mathrm{p}_{\mathrm{k}, \infty}=0, \mathrm{k} \neq 1\right)$. It is worth to mention that when all constituents are ideal gases, the Dalton's law of ideal gas mixtures is recovered (Chiapolino et al., 2017). Therefore, System (3.1) with thermodynamic closure Eq. (3.3) can be used for single phase flows and two-phase liquid-gas mixtures in mechanical and thermal equilibrium.

System (3.1) is hyperbolic with the sound speed given in Le Martelot et al. (2014) page 65. However this formula is quite complicated and useless, as the Wood (1930) sound speed is simpler and slightly greater than the thermal and mechanical equilibrium sound speed. The Wood speed of sound is consequently a better candidate for numerical computations, with respect to CFL computation as well as wave speeds computation in approximate Riemann solvers. It is given by:

$$
\frac{1}{\rho c^{2}}=\frac{1}{\sum_{k=1}^{N}\left[\frac{\alpha_{k}}{\rho_{k} c_{k}^{2}}\right]},
$$


where $\alpha_{k}=\frac{\rho Y_{k}}{\rho_{k}(T, p)}$ denotes the volume fraction of phase $k$.

In the limit of vanishing mass and volume fractions of the liquid phase and when a single gas constituent is present the Euler equations of gas dynamics are recovered. This remark enables building of a simple 1D reference solution to assess the accuracy of the various coupling methods.

\subsection{Reference solution}

An immersed piston in a fluid, here the air considered as an ideal gas, is set to motion impulsively at time $\mathrm{t}=0$. The impulsive motion to the right induces propagation of a right facing shock wave and a left facing expansion wave. A schematic (x,t) diagram is shown in Fig. 3.1 as well as qualitative profiles of velocity, pressure and density at a given time. This test problem is reminiscent of the exact Riemann problem solution except that the velocity between the two extreme waves is prescribed.

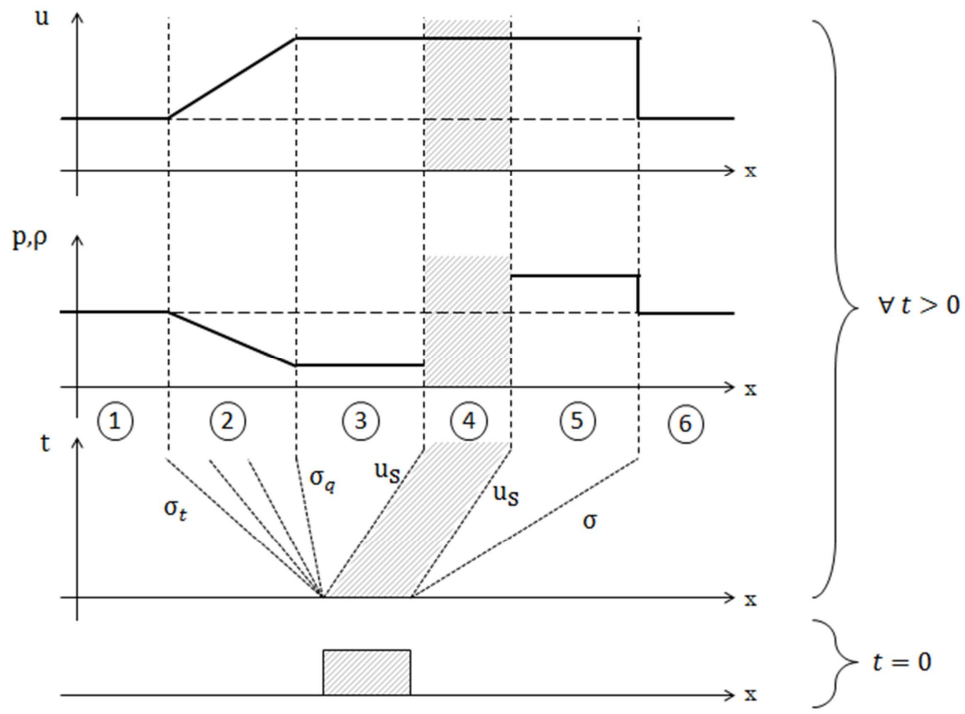

Figure 3.1- Immersed piston test problem. Schematic representation of the $(x, t)$ diagram and associated velocity, pressure and density profiles.

The various states present in the solution are:

- (1) left state initially at rest,

- (2) expansion wave,

- (3) fully expanded fluid,

- (4) piston,

- (5) post shock state,

- (6) right state initially at rest.

The exact solution is straightforward. Knowledge of the piston velocity combined to the RankineHugoniot relations determines fully state (5). The use of the Riemann invariants between state (1) and (3) where the velocity is the one of the piston determines fully state (3) and any point of the expansion wave (2). An example of such solution is given in Fig. 3.2 with initial data of Table 3.1.

\begin{tabular}{|c|c|}
\hline Temperatures $\mathrm{T}_{1}$ and $\mathrm{T}_{6}$ & $293 \mathrm{~K}$ \\
\hline Velocities $\mathrm{u}_{1}$ and $\mathrm{u}_{6}$ & 0 \\
\hline Pressures $\mathrm{p}_{1}$ and $\mathrm{p}_{6}$ & $100000 \mathrm{~Pa}$ \\
\hline Domain lenght & $1 \mathrm{~m}$ \\
\hline Initial position of the piston $(\mathrm{m})$ & $0.4 \leq \mathrm{x} \leq 0.5$ \\
\hline Velocity of the piston $\mathbf{u}_{\mathbf{s}}$ & $100 \mathrm{~m} / \mathrm{s}$ \\
\hline Final time & $1 \mathrm{~ms}$ \\
\hline
\end{tabular}

Table 3.1- Initial data for the immersed piston test problem. 
The air thermodynamics is modeled through EOS (3.2) with following data: $\gamma=1.4 ; \mathrm{p}_{\infty}=0 \mathrm{~Pa} ; \mathrm{q}=0 \mathrm{~J} / \mathrm{kg}$.

The corresponding exact solution is shown in Fig. 3.2. These results will serve as reference for the three coupling methods that are considered hereafter.
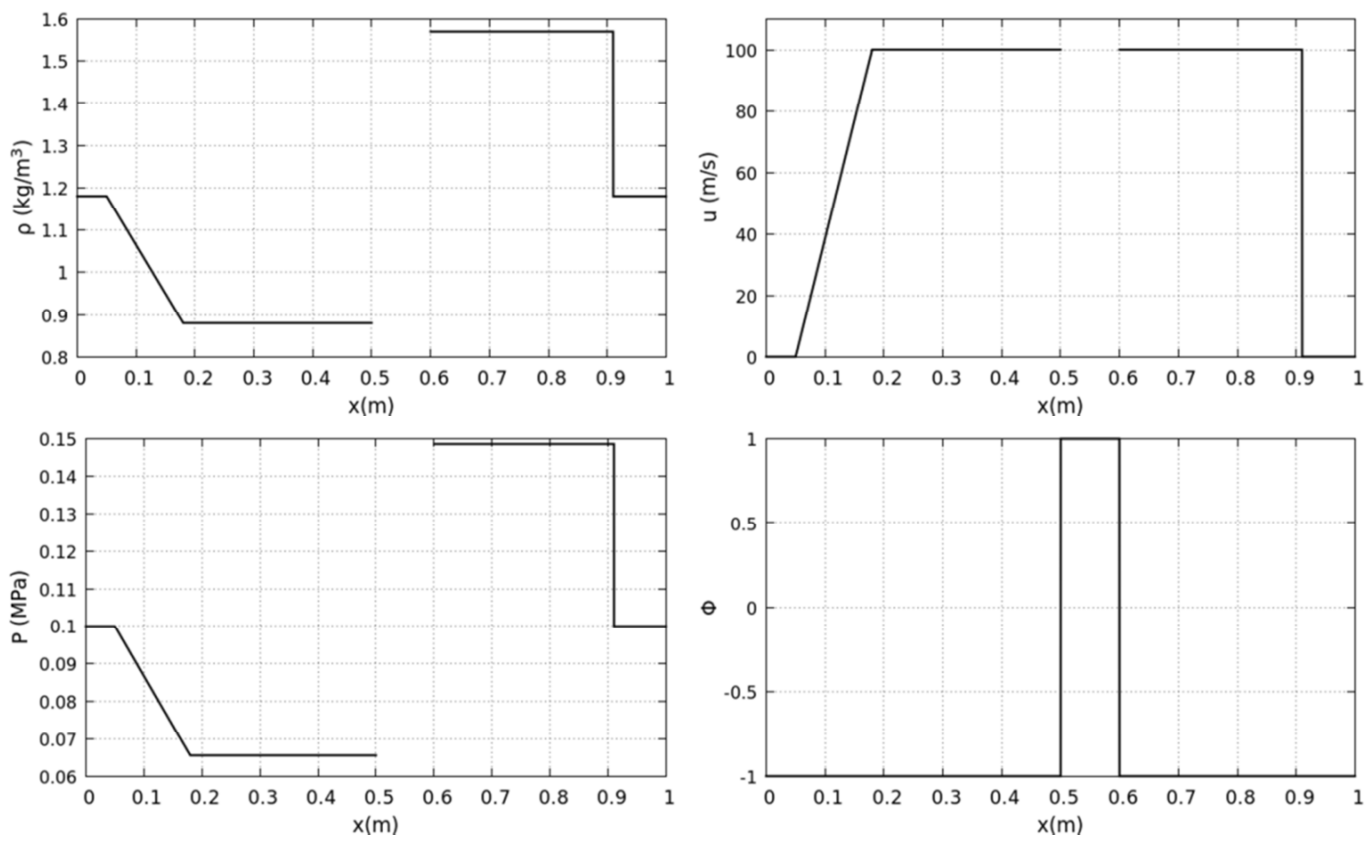

Figure 3.2- Exact solution for the immersed piston test problem moving in an ideal gas associated to initial data of Table 3.1.

\subsection{First coupling method: Velocity penalization}

In the present paragraph the coupling method is studied in 1D and the flow model (3.1) is reduced to the Euler equations to facilitate both presentation and comparison with the former exact solution. Also, a single moving rigid body is considered. The corresponding flow model with stiff velocity relaxation (penalization) among the body and fluid reads,

$$
\begin{aligned}
& \frac{\partial \rho}{\partial t}+\frac{\partial \rho u}{\partial x}=0 \\
& \frac{\partial \rho u}{\partial t}+\frac{\partial \rho u^{2}+p}{\partial x}=F_{\Phi} \\
& \frac{\partial \rho E}{\partial t}+\frac{\partial(\rho E+p) u}{\partial x}=F_{\Phi} \cdot u_{S} \\
& \frac{\partial \Phi}{\partial t}+\frac{\partial \Phi u_{S}}{\partial x}=0
\end{aligned}
$$

where $\mathrm{F}_{\Phi}$ represents the drag force exerted by the fluid on the solid and $\mathrm{F}_{\Phi_{\mathrm{S}}} \cdot \mathrm{u}_{\mathrm{S}}$ the power of this force.

The coupling force is modelled as,

$$
\mathrm{F}_{\Phi}=\rho \frac{1+\tilde{\Phi}}{2} \frac{\mathrm{u}_{\mathrm{S}}-\mathrm{u}}{\tau}
$$

where the relaxation time tends to zero $\left(\tau \rightarrow 0^{+}\right)$and factor $\frac{1+\tilde{\Phi}}{2}$ makes this force present in the numerical diffusion zone of the solid-fluid interface, on the solid side only. Similar approach is examined in Abgrall et al. (2014).

The modified Level-Set function $\tilde{\Phi}$ is defined as: 


$$
\tilde{\Phi}=\left\{\begin{array}{lll}
+1 & \text { if } & \Phi>0 \\
-1 & \text { if } & \Phi<0
\end{array}\right.
$$

This model is thermodynamically consistent as the entropy equation reads,

$$
\frac{\partial \rho s}{\partial t}+\frac{\partial \rho s u}{\partial x}=\rho \frac{1+\tilde{\Phi}}{2} \frac{\left(u_{s}-u\right)^{2}}{\tau} \geq 0
$$

In the stiff velocity relaxation limit, the production term vanishes, rendering the coupling method isentropic.

System (3.6) is solved by a splitting method, where the hyperbolic part is first solved with a MUSCL type scheme in the absence of source terms. The HLLC approximate Riemann solver of Toro et al. (1994) is used in all computations of the paper, to solve System (3.6) and its multi-D extension, System (3.1). The same equations are solved everywhere and the initial fluid state is set in the rigid body, except regarding the velocity, set to the one of the solid body. During this step, the Overbee limiter is used for the LevelSet function and another limiter (Minmod for example) is used for the other flow variables.

Second, the following ODE system is considered:

$$
\begin{aligned}
& \frac{\partial \rho}{\partial \mathrm{t}}=0 \\
& \frac{\partial \rho \mathrm{u}}{\partial \mathrm{t}}=\rho \frac{1+\tilde{\Phi}}{2} \frac{\mathrm{u}_{\mathrm{S}}-\mathrm{u}}{\tau} \\
& \frac{\partial \rho \mathrm{E}}{\partial \mathrm{t}}=\rho \frac{1+\tilde{\Phi}}{2} \frac{\mathrm{u}_{\mathrm{S}}-\mathrm{u}}{\tau} \cdot \mathrm{u}_{\mathrm{S}} \\
& \frac{\partial \Phi}{\partial \mathrm{t}}=0
\end{aligned}
$$

Rather than solving explicitly this ODE system, its asymptotic solution can be obtained easily as,

$\rho=\rho^{0} ; \Phi=\Phi^{0} ; \mathrm{u}=\mathrm{u}_{\mathrm{s}}$,

where the superscript ' 0 ' denotes the variables determined at the end of the hyperbolic step. Update of the total energy only requires specific attention.

Manipulating the equations of System (3.10), the internal energy equation is obtained as,

$$
\frac{\partial \mathrm{e}}{\partial \mathrm{t}}=\frac{1+\tilde{\Phi}}{2} \frac{\left(\mathrm{u}_{\mathrm{s}}-\mathrm{u}\right)^{2}}{\tau}
$$

As $\tau \rightarrow 0^{+}$, asymptotic expansion of the source terms shows immediately, as for the entropy equation, that,

$\mathrm{e}=\mathrm{e}^{0}$.

But as the velocity has been reset to the rigid body one, as expressed by Eq. (3.11), the total energy has to be corrected as,

$$
(\rho E)=\rho^{0}\left(e^{0}+\frac{1}{2} u_{s}^{2}\right)
$$

The coupling method thus consists in the reset of the velocity and the total energy with the help of Eqs. (3.11) and (3.12) in zones where $\Phi>0$. This method is consequently particularly simple. It is tested in Fig. 3.3 on the immersed piston test case of Figs. 3.1-3.2. 

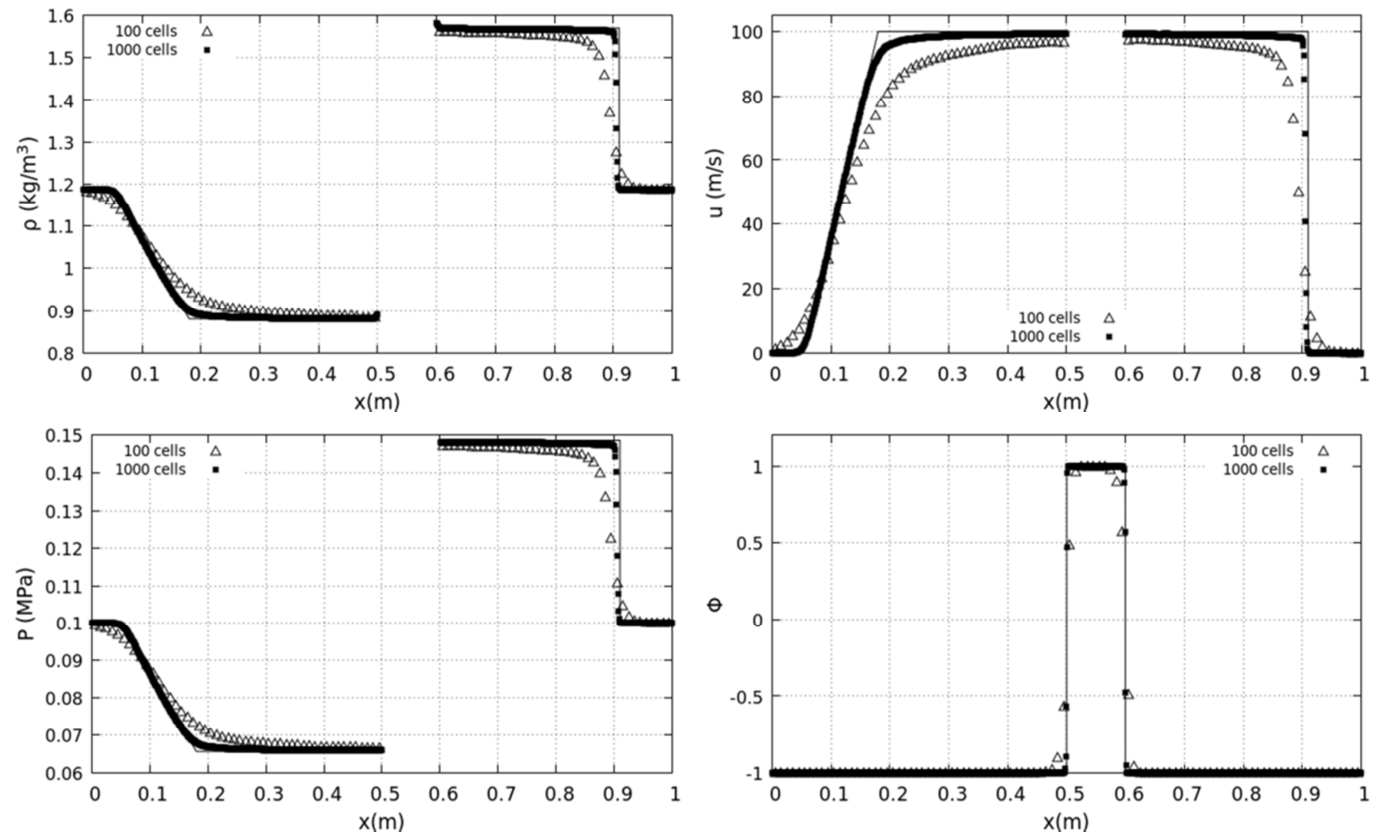

Figure 3.3- Relaxation method results. The first coupling method is used to compute the immersed piston test problem with two meshes, 100 and 1000 cells respectively and $C F L=0.9$. The Level-Set function is updated with the MUSCL-Overbee method while Minmod is used for the other variables. Numerical results are compared to the exact ones reported here in lines. Poor accuracy is obtained with 100 cells but the method tends to converge to the exact solution under mesh refinement.

This method converges to the exact solution, but the convergence rate seems slow. Another method, closer to the Ghost-Fluid-Method of Fedkiw et al. (1999) is thus addressed to improve convergence and efficiency.

\section{4 - Second coupling method: Ghost-Cell-type method}

In this second method, the hyperbolic step is unchanged and based on MUSCL type scheme with two limiters, as mentioned above. As in all computations the HLLC solver is used in the hyperbolic step. The coupling step is based on extrapolated variables from the fluid to the solid:

$$
\left(\begin{array}{l}
\rho_{i} \\
u_{i} \\
p_{i}
\end{array}\right)=\left(\begin{array}{l}
\rho_{j} \\
u_{s} \\
p_{j}
\end{array}\right) \text { if }\left\{\begin{array}{l}
\Phi_{i}^{0} \geq 0 \\
\Phi_{j}^{0}<0
\end{array}\right.
$$

where $i$ and $j$ denote two neighboring cells, $i$ being in the solid body and $j$ in the fluid.

In Ghost-Cell (GC) methods the band of cells in which the extrapolation is done has importance. When extrapolation is done with System (3.13), interfacial cells only are corrected. But at the next time step the interface may leave the cell and enter another cell occupied formerly by the solid. This cell must consequently be filled with a consistent set of variables. This issue is illustrated in the Fig. 3.4.

Let us denote by $\mathrm{W}$ the set of primitive variables used during the extrapolation $\mathrm{W}=(\rho, \mathrm{u}, \mathrm{p})^{\mathrm{T}}$ and $\mathrm{U}$ the associated set of conservative variables. The extrapolation is done in the solid cell on the graph on top at left. No precise state is prescribed in cell $i+2$. Then the Riemann problem is solved everywhere (graph at bottom) and during the time step, the interface changes cell. At the end of the time step cell $i+1$ is now a fluid cell but the state it contains is wrong, as the Riemann problem solution between cells $i+1$ and $i+2$ is wrong too. Therefore, at the next time step, when extrapolation is done from cell $i+1$ to cell $i+2$, a wrong state is copied and the solution diverges. 

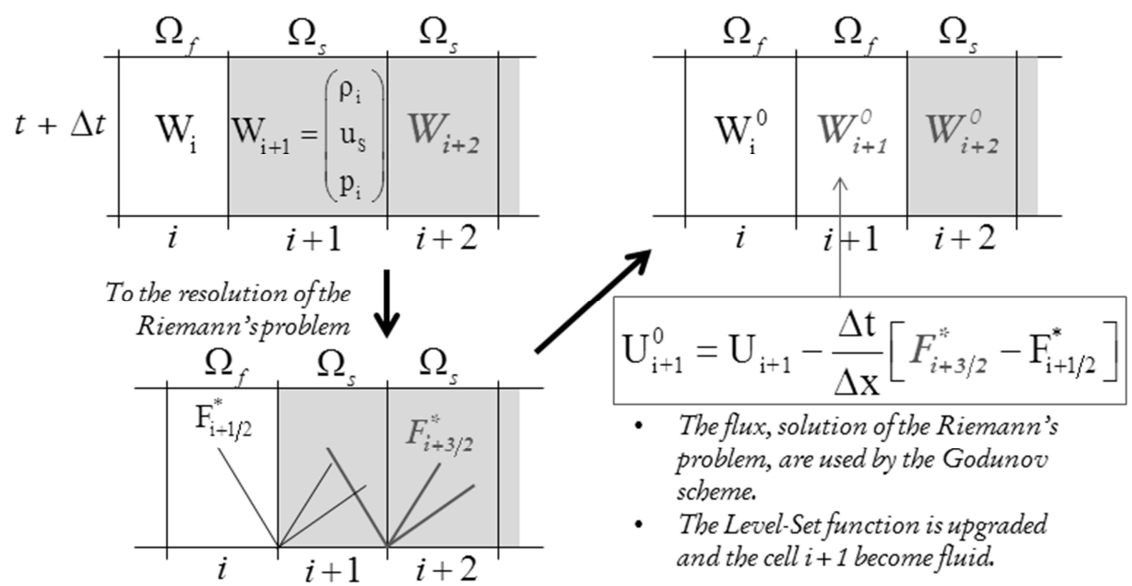

- The flux, solution of the Riemann's problem, are used by the Godunov scheme.

- The Level-Set function is upgraded and the cell $i+1$ become fluid.

Figure 3.4- Schematic representation of the numerical pollution occurring when the extrapolation is done in a too narrow band of cells.

Typical results obtained with this numerical pollution effect are shown in the Fig. 3.5 where the same immersed piston test problem as before is rerun. Only the velocity graph is shown for the sake of conciseness.

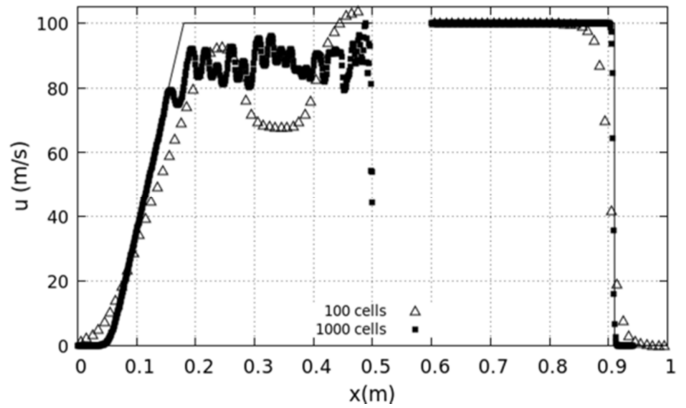

Figure 3.5- Illustration of the numerical pollution effect when a too narrow band of Ghost cells is used for extrapolation. The piston velocity is transmitted to the fluid on the right side but the expansion wave on the left side is wrong. This issue persists when the mesh is refined.

This issue is well known in the literature (Liu et al., 2003, 2006, 2008). Following these references the extrapolation method given by System (3.13) is extended to a band of two cells in the solid in the vicinity of the interface. The corrected algorithm is summarized in System (3.14):

$$
\left\{\begin{array}{l}
\left(\begin{array}{l}
\rho_{\mathrm{i}} \\
u_{\mathrm{i}} \\
p_{\mathrm{i}}
\end{array}\right)=\left(\begin{array}{l}
\rho_{\mathrm{j}} \\
\mathrm{u}_{\mathrm{s}} \\
p_{\mathrm{j}}
\end{array}\right) \text { if }\left\{\begin{array}{l}
\Phi_{\mathrm{i}}^{0} \geq 0 \\
\Phi_{\mathrm{j}}^{0}<0
\end{array}\right. \\
\left(\begin{array}{l}
\rho_{\mathrm{ii}} \\
\mathrm{u}_{\mathrm{ii}} \\
p_{\mathrm{ii}}
\end{array}\right)=\left(\begin{array}{l}
\rho_{\mathrm{j}} \\
u_{\mathrm{s}} \\
p_{\mathrm{j}}
\end{array}\right) \text { if }\left\{\begin{array}{l}
\Phi_{\mathrm{ii}}^{0} \geq 0 \\
\Phi_{\mathrm{j}}^{0}<0
\end{array}\right.
\end{array}\right.
$$

Where $i$ denotes the first solid cell in contact with the fluid cell $j$ and ii the second solid cell, neighboring cell i. With this correction the immersed piston test is rerun and the results of Fig. 3.6 are obtained. 

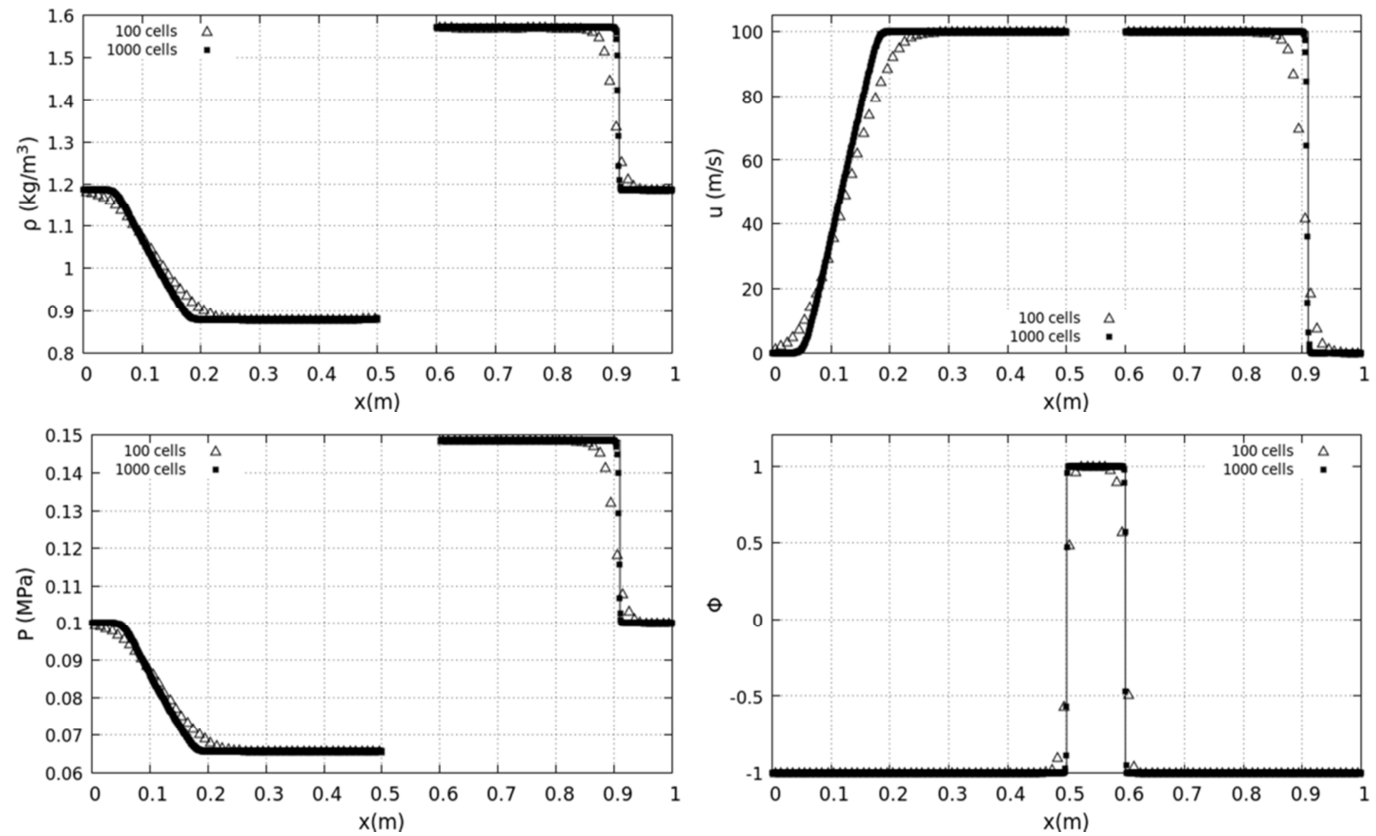

Figure 3.6- Ghost-Cell method with extended band of cells - Results for the immersed piston test. Two meshes are used with 100 and 1000 points respectively. The time step fulfills CFL $=0.9$. The MUSCL scheme with Overbee is used for the Level-Set transport and Minmod is used for the other flow variables. The method converges to the exact solution shown in full lines under mesh refinement. With 100 cells, the accuracy is better than with the former relaxation method.

With the Ghost-Cell method summarized in System (3.14) interface conditions are matched for the two meshes considered. However, regarding the coarser one (100 cells) the shock is delayed compared to the exact solution of Fig. 3.2. The method improves efficiency compared to the relaxation one, but seems still perfectible. A refined version is examined in the next paragraph.

\section{5 - Third coupling method: Ghost-Cell-type method with improved velocity extrapolation}

The same Ghost-Cell-type method as before is considered, as summarized by System (3.14) but the extrapolated state is reconsidered in the aim of convergence improvement.

The present approach follows conventional method for the treatment of piston boundary conditions and avoids local resolution of exact or approximate Riemann problem, as done for example in Liu et al. (2006) and Farhat et al. (2012). It is thus aimed to simplify the related methods and facilitate coupling with more sophisticated flow models, as stated in the Introduction.

Let us consider a fluid at right and a solid at left. To mimic piston motion at prescribed velocity $\mathrm{u}^{*}=\mathrm{u}_{\text {piston }}$, where $\mathrm{u}^{*}$ denotes solution of the Riemann problem between two fluid states at left and at right, a fictitious state at left has to be determined. In this approach, the star state solution corresponds to the prescribed piston velocity. This approach is schematized in Fig. 3.7.

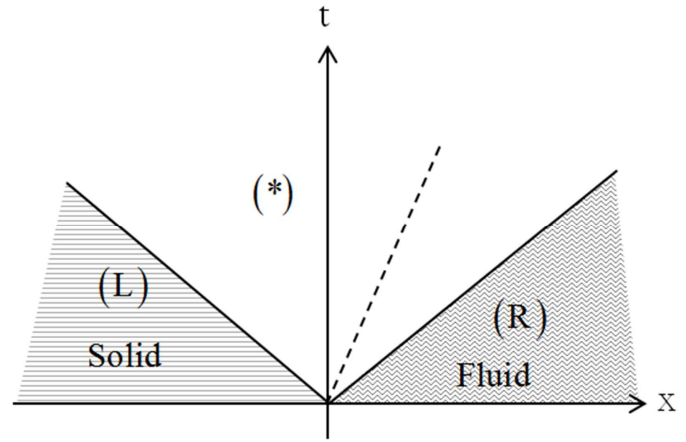

Figure 3.7- Schematic representation of the 'inverse' Riemann problem solved at the interface. The left state has to be determined in order that the star velocity becomes the one of the rigid body.

The right state (R) being known the left state $(\mathrm{L})$ has to be determined in order that $\mathrm{u}^{*}=\mathrm{u}_{\text {piston }}$. 
For the sake of simplicity in the analysis the approximate acoustic solver is considered:

$$
\left\{\begin{array}{l}
\mathrm{P}_{\mathrm{L}}+\mathrm{Z}_{\mathrm{L}} \mathrm{u}_{\mathrm{L}}=\mathrm{P}^{*}+\mathrm{Z}_{\mathrm{L}} \mathrm{u}^{*} \\
\mathrm{P}_{\mathrm{R}}-\mathrm{Z}_{\mathrm{R}} \mathrm{u}_{\mathrm{R}}=\mathrm{P}^{*}-\mathrm{Z}_{\mathrm{R}} \mathrm{u}^{*}
\end{array}\right.
$$

The pressure and density in the left state are assumed extrapolated from the right state, as done before with the former Ghost-Cell method. Consequently,

$\mathrm{P}_{\mathrm{R}}=\mathrm{P}_{\mathrm{L}}=\mathrm{P} ; \mathrm{Z}_{\mathrm{L}}=\mathrm{Z}_{\mathrm{R}}=\mathrm{Z}$

The Riemann problem solution thus reads,

$$
\mathrm{u}^{*}=\frac{\mathrm{u}_{\mathrm{L}}-\mathrm{u}_{\mathrm{R}}}{2} ; \mathrm{P}^{*}=\mathrm{P}+\frac{\mathrm{Z}\left(\mathrm{u}_{\mathrm{L}}-\mathrm{u}_{\mathrm{R}}\right)}{2}
$$

As $\mathrm{u}^{*}=\mathrm{u}_{\text {piston }}$ the left state velocity is determined as,

$\mathrm{u}_{\mathrm{L}}=2 \mathrm{u}_{\text {piston }}-\mathrm{u}_{\mathrm{R}}$.

It also appears that the pressure at the rigid body surface is,

$$
\mathrm{P}^{*}=\mathrm{P}+\mathrm{Z}\left(\mathrm{u}_{\text {piston }}-\mathrm{u}_{\mathrm{R}}\right) \text {. }
$$

The term $\mathrm{Z}\left(\mathrm{u}_{\text {piston }}-\mathrm{u}_{\mathrm{R}}\right)$ induces compression or expansion depending on the sign of the velocity difference. In any case it anticipates shock or expansion appearance in the sense that it corresponds to the pressure, solution of the Riemann problem at the interface. However, there is no need to solve explicitly the Riemann problem locally. The HLLC solver (or any other flow solver) used to update the hyperbolic model will compute correctly the star pressure.

Thanks to this correction, the extrapolation method, analogue of System (3.14) now reads,

$$
\left\{\begin{array}{l}
\left(\begin{array}{l}
\rho_{\mathrm{i}} \\
\mathrm{u}_{\mathrm{i}} \\
\mathrm{p}_{\mathrm{i}}
\end{array}\right)=\left(\begin{array}{c}
\rho_{\mathrm{j}} \\
2 \mathrm{u}_{\mathrm{s}}-\mathrm{u}_{\mathrm{j}} \\
\mathrm{p}_{\mathrm{j}}
\end{array}\right) \text { if }\left\{\begin{array}{l}
\Phi_{\mathrm{i}}^{0} \geq 0 \\
\Phi_{\mathrm{j}}^{0}<0
\end{array}\right. \\
\left(\begin{array}{c}
\rho_{\mathrm{ii}} \\
\mathrm{u}_{\mathrm{ii}} \\
\mathrm{p}_{\mathrm{ii}}
\end{array}\right)=\left(\begin{array}{c}
\rho_{\mathrm{j}} \\
2 \mathrm{u}_{\mathrm{s}}-\mathrm{u}_{\mathrm{j}} \\
\mathrm{p}_{\mathrm{j}}
\end{array}\right) \text { if }\left\{\begin{array}{c}
\Phi_{\mathrm{ii}}^{0} \geq 0 \\
\Phi_{\mathrm{j}}^{0}<0
\end{array}\right.
\end{array}\right.
$$

As before, $i$ denotes the first solid cell in contact with the fluid cell $j$ and ii the second solid cell, neighboring cell $\mathrm{i}$. The immersed piston test problem is now rerun with this modification. Corresponding results are shown in Fig. 3.8.
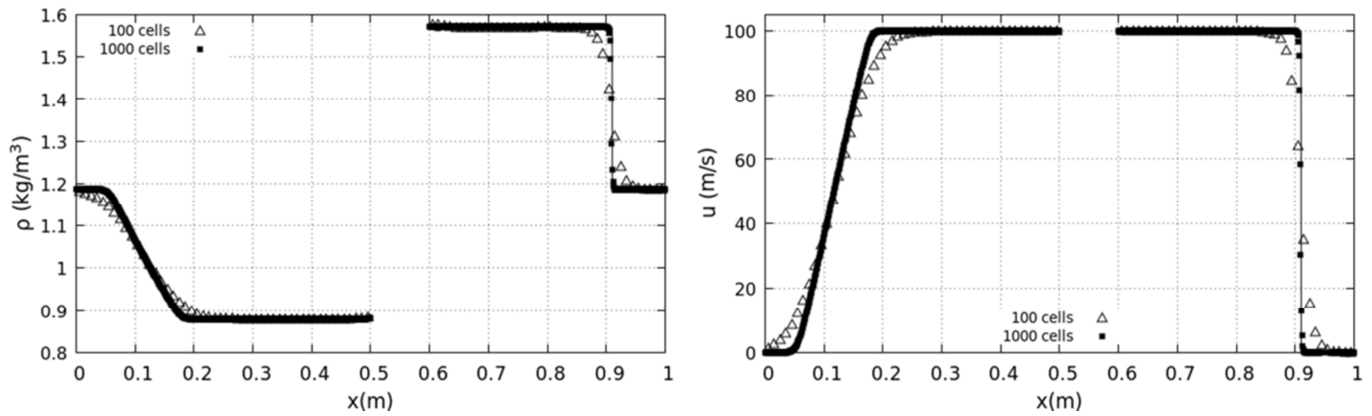

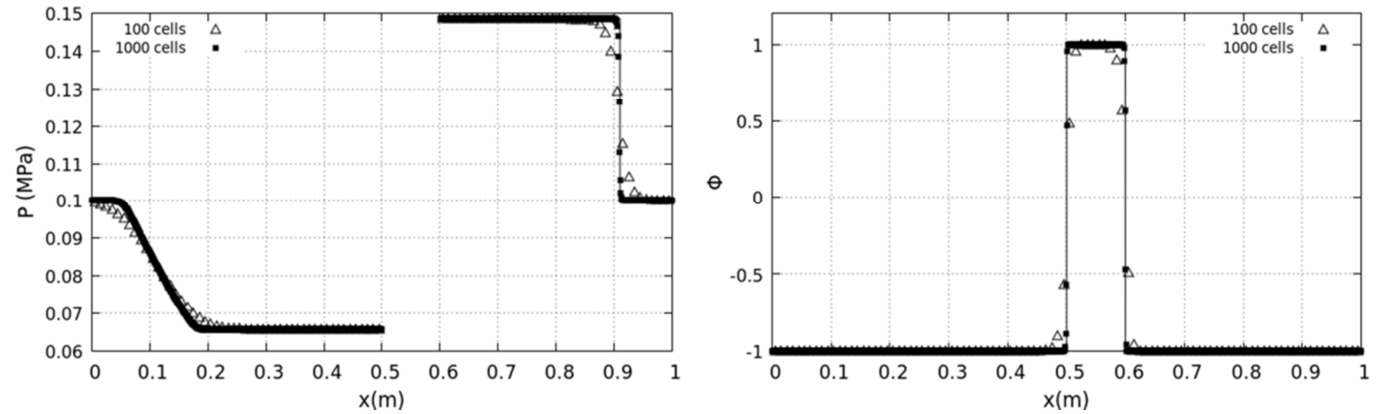

Figure 3.8- Ghost-Cell method with extended band of cells and modified boundary conditions - Results for the immersed piston test. Two meshes are used with 100 and 1000 points respectively. The time step fulfills CFL $=0.9$. The MUSCL scheme with Overbee is used for the Level-Set transport and Minmod is used for the other flow variables. The method converges to the exact solution shown in full lines under mesh refinement. With 100 cells, the shock position is now correct.

The three coupling methods are now compared on the same graph with a coarse mesh involving 100 cells. Corresponding results are shown in Fig. 3.9.
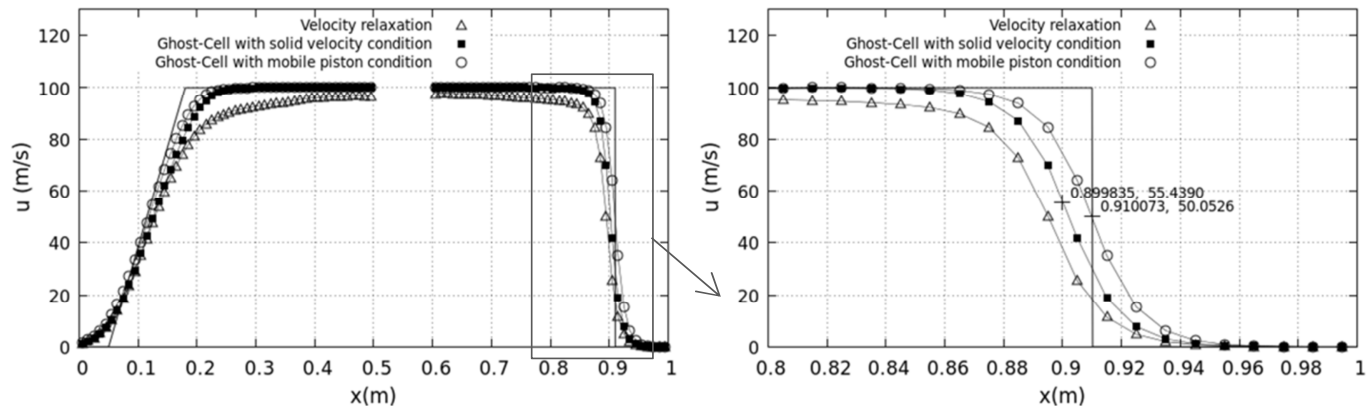

Figure 3.9- Comparison of the various coupling methods with 100 cells against the exact solution. The modified Ghost-Cell method with piston boundary condition improves the results.

We have shown that the three methods converge to the exact solution, but the Ghost-Cell method with modified boundary condition improves the convergence rate. We now address extension of this last method in multi-D on unstructured meshes.

\section{4-Multidimensional extension}

The coupling method is now extended to multi-D. Transport of the Level-Set function in multi-D follows the lines of Chiapolino et al. (2017) regarding the volume fraction transport of their diffuse interface flow model. Therefore, it is not detailed anymore.

\subsection{Solid-fluid coupling method}

Mixed cells have to be defined and to do this solid cells have to be defined first. As already mentioned a cell is considered solid when the Level-Set function $\Phi$ is positive at the cell center and fluid otherwise. It becomes a mixed cell when one of its direct neighbors has $\Phi$ with opposite sign, as shown in Fig. 4.1. It is worth mentioning that none of the fluid cells $(\Phi<0)$ are considered as mixed and are solved with the hyperbolic solver routinely. Thus mixed cells are defined as solid one $(\Phi>0)$ that share an edge with at least one fluid cell $(\Phi<0)$. 


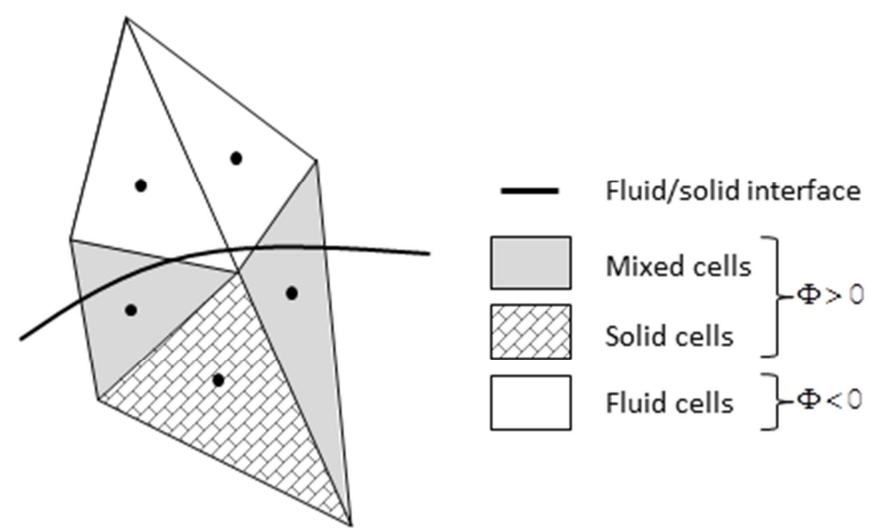

Figure 4.1- Schematic representation of the interface and mixed cells.

2D extension of the Ghost-Cell method proceeds in two-steps.

a) Approximation of the fluid state in mixed cells

When a mixed cell is detected the fluid state in the corresponding cell is computed as a volume average of the neighboring fluid cells. This average is obviously based on conservative variables.

Let's define $\Omega_{\mathrm{vf}}$ the set of neighboring fluid cells of the considered mixed cell. The set of conservative variable in the mixed cell is obtained as,

$\overline{\mathbf{U}}=\frac{\sum_{\mathrm{k} \in \Omega_{\mathrm{vf}}} \mathrm{V}_{\mathrm{k}} \mathrm{U}_{\mathrm{k}}}{\sum_{\mathrm{k} \in \Omega_{\mathrm{vf}}} \mathrm{V}_{\mathrm{k}}}$,

where $V_{k}$ represents the volume of cell $\mathrm{k}$. From this vector of conservative variables, primitive ones are deduced:

$\widetilde{\mathrm{W}}=(\tilde{\rho}, \tilde{\mathrm{u}}, \tilde{\mathrm{v}}, \tilde{\mathrm{p}})^{\mathrm{T}}$

Symbol $\sim$ is used to make distinction with the volume average symbol.

These primitive variables are used during the extrapolation step that follows.

b) Extrapolation across the interface

The same piston boundary conditions are used in the direction normal to the solid-fluid interface. As the flow model (3.1) is inviscid the tangential velocity has to be extrapolated as well.

Let's consider two cells having a common edge and having Level-Set functions of different signs. Necessarily the interface crosses the line segment connecting the two cell centers, as shown in Fig. 4.2 where the cell center containing a fluid state is denoted by $\mathrm{F}$, the one containing a solid is denoted by $\mathrm{G}$ and the interface point is denoted by $\mathrm{S}$. 


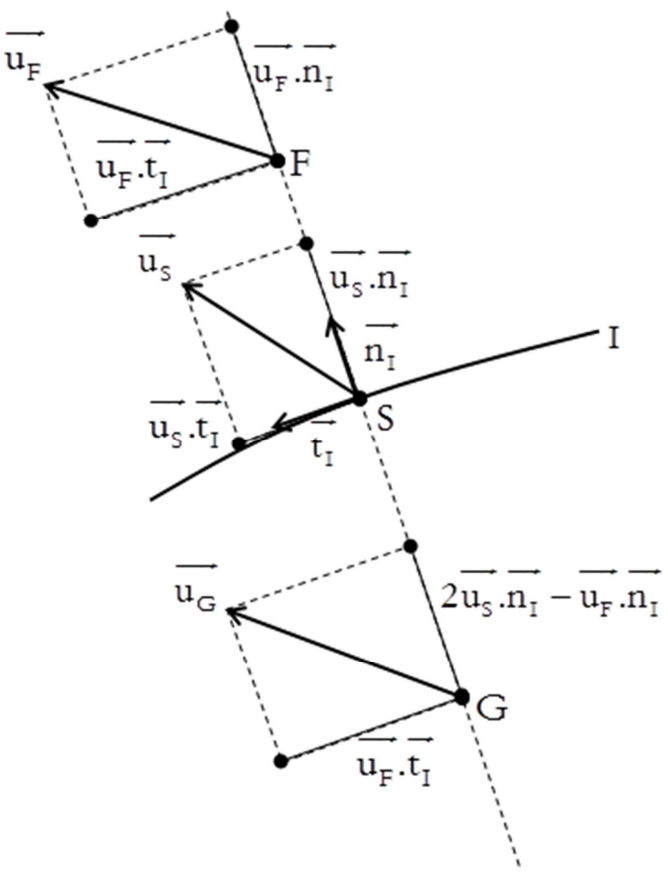

Figure 4.2- Schematic representation of two mesh points having a common edge and Level-Set functions of different signs, positive for the solid point $G$ and negative for the fluid point $F$.

The normal vector to the interface is defined as,

$$
\overrightarrow{\mathrm{n}_{\Phi}}=-\frac{\vec{\nabla} \Phi}{\|\vec{\nabla} \Phi\|}
$$

where $\vec{\nabla} \Phi$ is computed in each cell as mentioned in Section 2 with the least-square method and extended set of neighbors. The normal vector used in the extrapolation procedure is the one computed in the mixed cell, as defined earlier.

The velocity components are extrapolated in the solid as,

$\overrightarrow{\mathrm{u}_{\mathrm{G}}} \cdot \overrightarrow{\mathrm{n}_{\mathrm{I}}}=2 \overrightarrow{\mathrm{u}_{\mathrm{S}}} \cdot \overrightarrow{\mathrm{n}_{\mathrm{I}}}-\overrightarrow{\mathrm{u}_{\mathrm{F}}} \cdot \overrightarrow{\mathrm{n}_{\mathrm{I}}}$ and $\overrightarrow{\mathrm{u}_{\mathrm{G}}} \cdot \overrightarrow{\mathrm{u}_{\mathrm{I}}}=\overrightarrow{\mathrm{u}_{\mathrm{F}}} \cdot \overrightarrow{\mathrm{t}_{\mathrm{I}}}$

along the normal and tangential directions respectively.

The rest of the primitive variable vector is copied in the solid cell.

\section{c) Extrapolation to the Ghost-Cell band}

As previously mentioned in the $1 \mathrm{D}$ case and comments related to Fig. 3.5 additional cells need to be filled with consistent fluid states. Indeed, at the next time step the interface may move away from the current cell and a solid cell may become a fluid one. The extrapolation procedure is extended to a wider stencil to anticipate appearance of extra fluid cells. The 'additional cells' where a fluid state has to be defined are shown in Fig. 4.3. They are defined as solid cells having a mixed cell as direct neighbor.

A conservative average is performed with the neighboring mixed cells (also defined in Fig. 4.3), that have been updated with the sequence (4.1-4.2-4.3).

An extra conservative average is done on the mixed cells with the same definitions as Eqs. (4.1)(4.2) where the mixed-cells volumes and states are used instead of the fluid ones. The resulting volume average done with the mixed cells is used to update the 'additional cells'.

Two sequences of volume averages are consequently done with the present method:

- First with the fluid cells to update the mixture cells;

- Second with the mixture cells to update the 'additional cells'. 
Between these two averaging steps the piston boundary condition (4.3) is obviously used.

This simple averaging-extrapolation method simplifies significantly existing solid-fluid coupling methods on unstructured meshes.
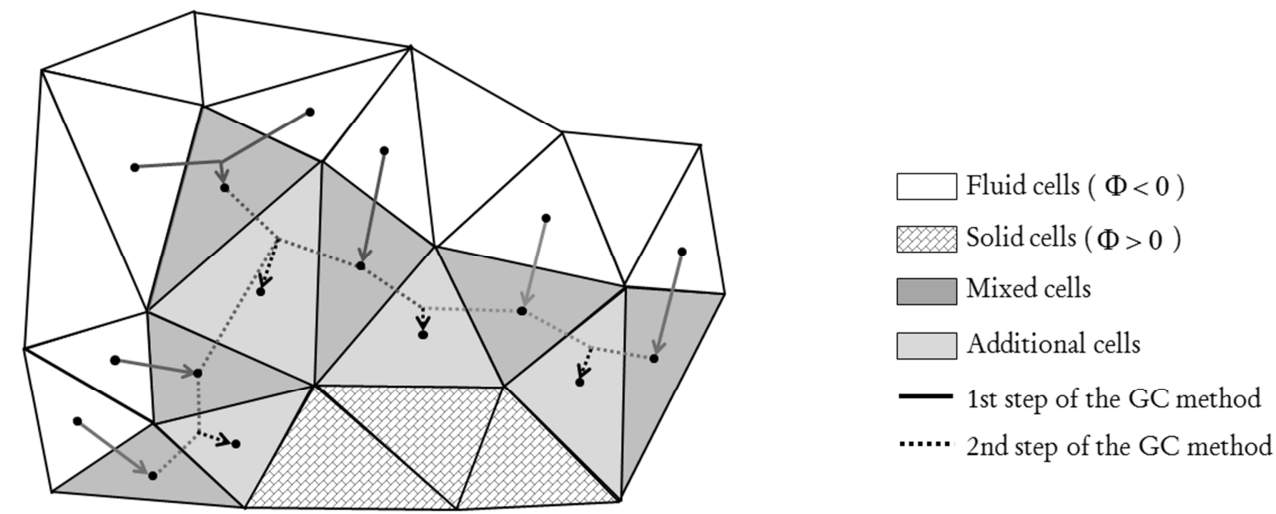

Figure 4.3 - Treatment of the Ghost-Cell band in 2D. All grey cells and hatched ones represent solid cells. These solid cells are divided in 3 categories. The first one, in dark grey, corresponds to the mixed cells in which extrapolation is done following (4.3) after volume average (4.1)-(4.2). The second layer, represented in grey, corresponds to the solid direct neighbors of the mixed cells, as defined in Figure 3.4. Fluid state is set in these cells by averaging conservative variables of the surrounding mixed cells with the help of (4.1)-(4.2) definitions. States and volumes of mixed-cells are used in these formulas. The third one with hatched symbols corresponds to the solid cells that are not modified by the coupling method.

To examine accuracy of the method reference results are determined, as detailed in the next subsection.

\subsection{Reference solution}

The reference solution is obtained by considering a supersonic two-phase flow around a rigid body at rest, as depicted in Fig. 4.4.

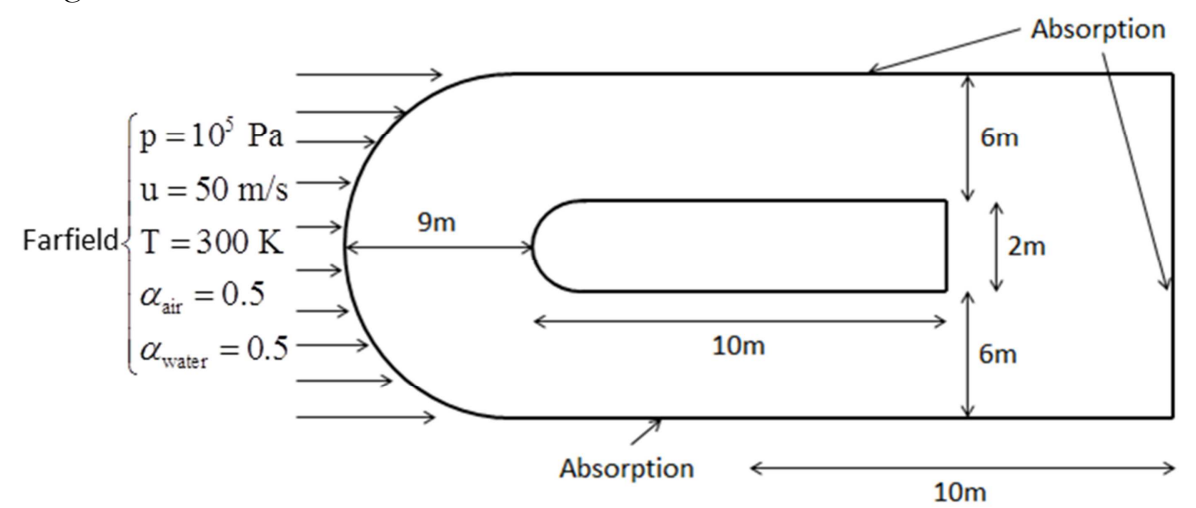

Figure 4.4- Reference 2D computations - A supersonic two-phase mixture of liquid water and air flows around a rigid blunt body at rest.

The various dimensions of the rigid body and computational domain are shown in Fig. 4.4. The reference results are obtained with the MUSCL type scheme already mentioned, with Minmod limiter and with a mesh density corresponding to an average space size of $3 \mathrm{~cm}$.

The various parameters of the equations of state, used in Eqs. (3.2) and (3.3), are given hereafter,

$$
\left\{\begin{array}{l}
\gamma_{\text {air }}=1.4 ; \mathrm{p}_{\infty, \text { air }}=0 \mathrm{~Pa} ; \mathrm{q}_{\text {air }}=0 \mathrm{~J} / \mathrm{kg} ; \mathrm{C}_{\mathrm{v}, \text { air }}=719 \mathrm{~J} / \mathrm{kg} / \mathrm{K} \\
\gamma_{\text {water }}=2.35 ; \mathrm{p}_{\infty, \text { water }}=10^{9} \mathrm{~Pa} ; \mathrm{q}_{\text {water }}=-1167 \mathrm{~kJ} / \mathrm{kg} ; \mathrm{C}_{\mathrm{v}, \text { water }}=1816 \mathrm{~J} / \mathrm{kg} / \mathrm{K}
\end{array}\right.
$$

The corresponding steady state solution is shown in Fig. 4.5. 


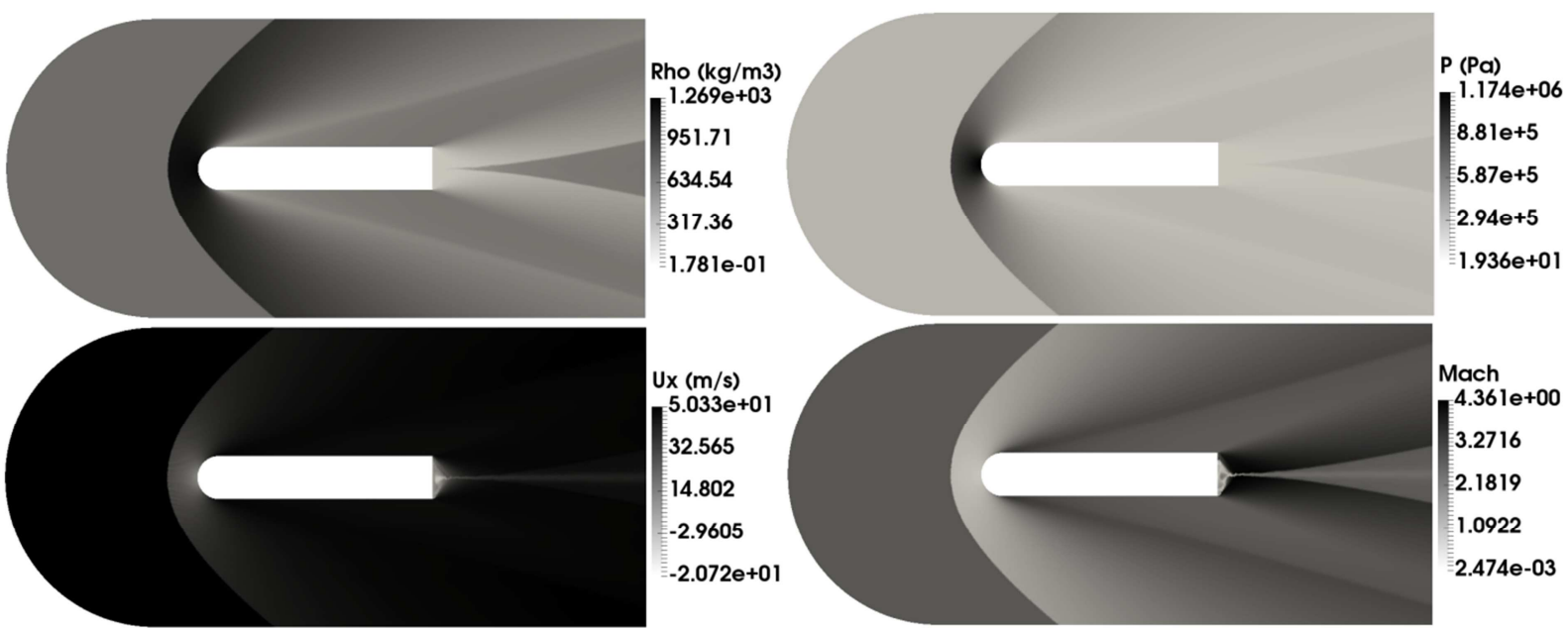

Figure 4.5- Supersonic two-phase test problem: Steady state results. As a consequence of low mixture sound speed (3.5), the flow entering at velocity $50 \mathrm{~m} / \mathrm{s}$ is supersonic. The results are obtained with MUSCL scheme and Minmod limiter on a mesh involving 280786 triangular cells and CFL $=0.9$. In these computations a wall boundary condition is used at the body surface while supersonic state is prescribed at the inlet. An absorption condition is prescribed at the other boundary surfaces.

A detached shock wave is clearly visible in front of the obstacle as well as an expansion zone in the rear. The same situation is now addressed with a fluid initially at rest and moving solid body.

\subsection{Validation}

Similar flow situation as the one of Fig. 4.4 is now considered. The coupling method is used between the moving rigid body, tracked by the Level-Set method with Overbee limiter and the fluid flow, initially at rest in the present configuration. The various dimensions and geometrical data are shown in Fig. 4.6.

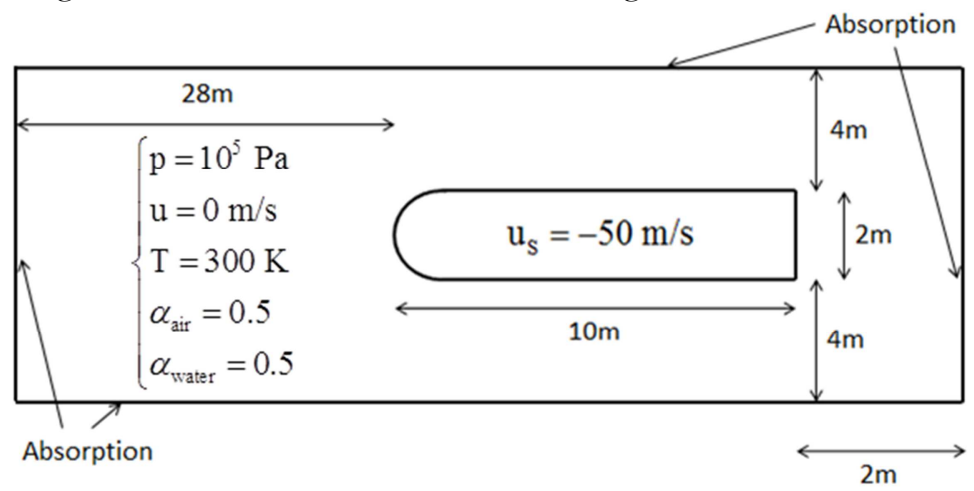

Figure 4.6- Configuration studied for the coupling method validation. A rigid blunt body moves at supersonic speed in the same two-phase mixture as the one entering at the inflow in Figs. 4.4 and 4.5.

The mesh density is the same as before, with an average space size of $3 \mathrm{~cm}$. The Minmod limiter is used for the various flow variables except the Level-Set used to track the rigid body, solved with MUSCL scheme with Overbee. Computed results are shown in Fig. 4.7 at time $0.5 \mathrm{~s}$, when steady state is reached. 


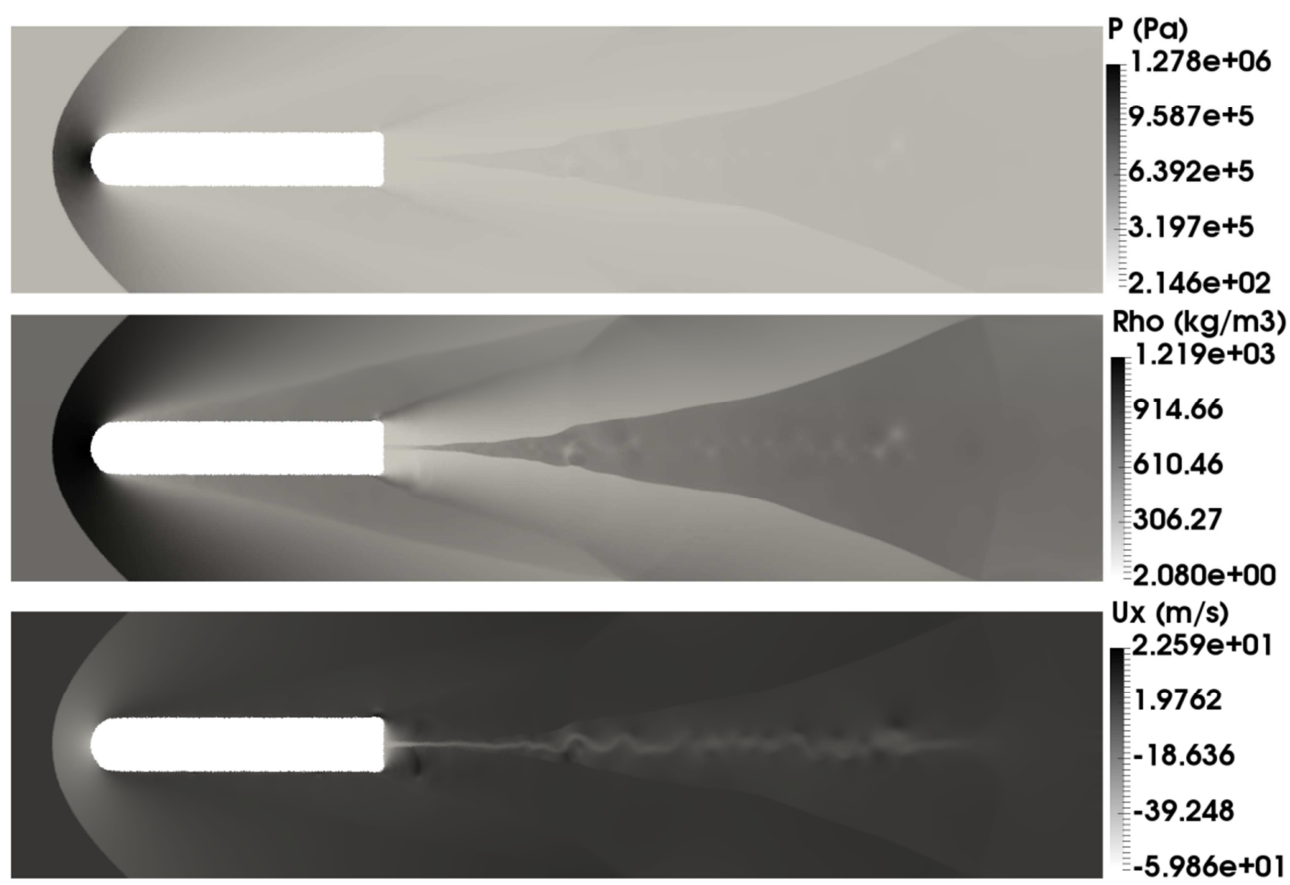

Figure 4.7- Computations of the moving body in two-phase mixture with the coupling method. Density, velocity and pressure contours are shown at time $0.5 \mathrm{~s}$ and computed with a mesh involving 302812 cells corresponding to an average space step of 3 $\mathrm{cm}$ and $\mathrm{CFL}=0.8$. The rigid body is tracked with the Level-Set function and Overbee limiter while the other flow variables are computed with the Minmod limiter and MUSCL scheme.

Computed results of Figs. 4.5 and 4.7 are in close agreement. First the same detached shock wave is visible as well as expansion zone at rear of the projectile. Second, the shape of the projectile is very well preserved by the Level-Set-Overbee method. Last, it is possible to address quantitative comparison by extracting flow variables from the two sets of results along a given direction, such as the Ox axis for instance. This is done in Fig. 4.8 for the pressure profiles.
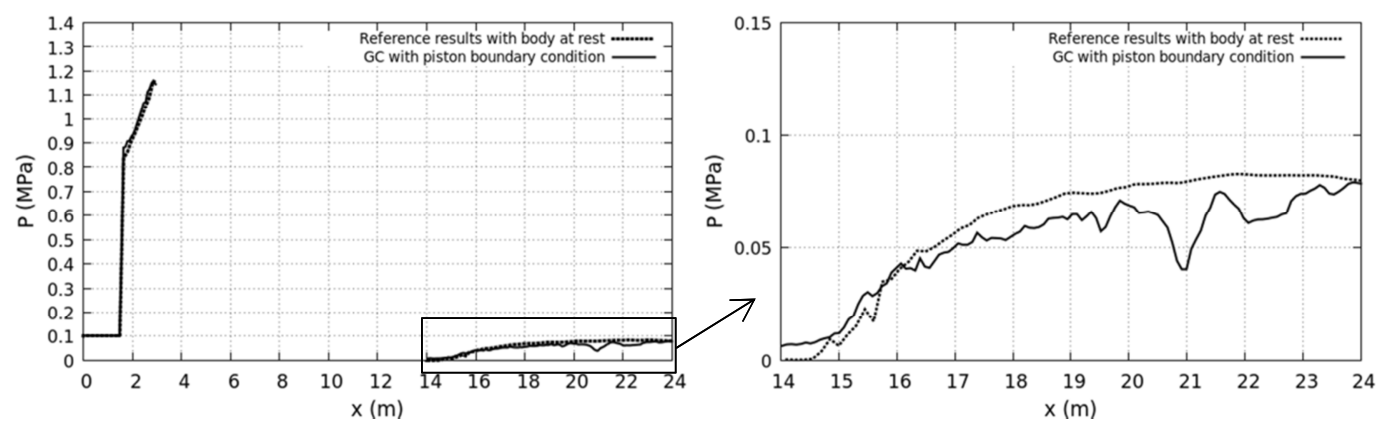

Figure 4.8-Comparison of the pressure profiles along the $\mathrm{Ox}$ axis with rigid body at rest and body fitted mesh associated to computations of Fig. 4.5 and the ones of Fig. 4.7 with moving body and present coupling method. Very good agreement is observed. Obviously some fluctuations are present due to some artificial rugosity effects.

The results of Fig. 4.8 confirm validity of the coupling method with comparable accuracy as the one observed in $1 \mathrm{D}$ in the Figures 3.8-3.9. Moreover, the method remains simple to implement. Illustrations of the method's capabilities in the frame of the two-phase flow model (3.1) are now addressed.

\subsection{Illustrations with two-phase flow effects}

Two projectiles of $5 \mathrm{~mm}$ radius with imposed velocities are considered and move at high velocity through air and impact a liquid water domain settled in the air. The air is considered as an ideal gas and the liquid is assumed governed by the stiffened gas EOS (3.2). As material interfaces between the various fluids are present the flow model (3.1) with mixture EOS (3.3) is an appropriate candidate, in particular when phase transition is considered (Saurel et al., 2016). Thermodynamic data of the various fluids are given hereafter: 


$$
\left\{\begin{array}{l}
\gamma_{\mathrm{w}, \text { liquid }}=2.35 ; \mathrm{p}_{\infty, \mathrm{w}, \text { liquid }}=10^{9} \mathrm{~Pa} ; \mathrm{q}_{\mathrm{w}, \text { liquid }}=-1167 \mathrm{~kJ} / \mathrm{kg} ; \mathrm{C}_{\mathrm{v}, \mathrm{w}, \text { liquid }}=1816 \mathrm{~J} / \mathrm{kg} / \mathrm{K} \\
\gamma_{\mathrm{w}, \text { vapor }}=1.43 ; \mathrm{p}_{\infty, \mathrm{w}, \text { vapor }}=0 \mathrm{~Pa} ; \mathrm{q}_{\mathrm{w}, \text { vapor }}=2030 \mathrm{~kJ} / \mathrm{kg} ; \mathrm{C}_{\mathrm{v}, \mathrm{w}, \text { vapor }}=1040 \mathrm{~J} / \mathrm{kg} / \mathrm{K} \\
\gamma_{\text {air }}=1.4 ; \mathrm{p}_{\infty, \text { air }}=0 \mathrm{~Pa} ; \mathrm{q}_{\text {air }}=0 \mathrm{~J} / \mathrm{kg} ; \mathrm{C}_{\mathrm{v}, \text { air }}=719 \mathrm{~J} / \mathrm{kg} / \mathrm{K}
\end{array}\right.
$$

Phase transition is considered through local thermodynamic equilibrium. Simple and fast thermochemical relaxation solver has been developed in Chiapolino et al. (2017) and is used in the present computations. Initial and boundary conditions are given in the Fig. 4.9 as well as geometrical data.

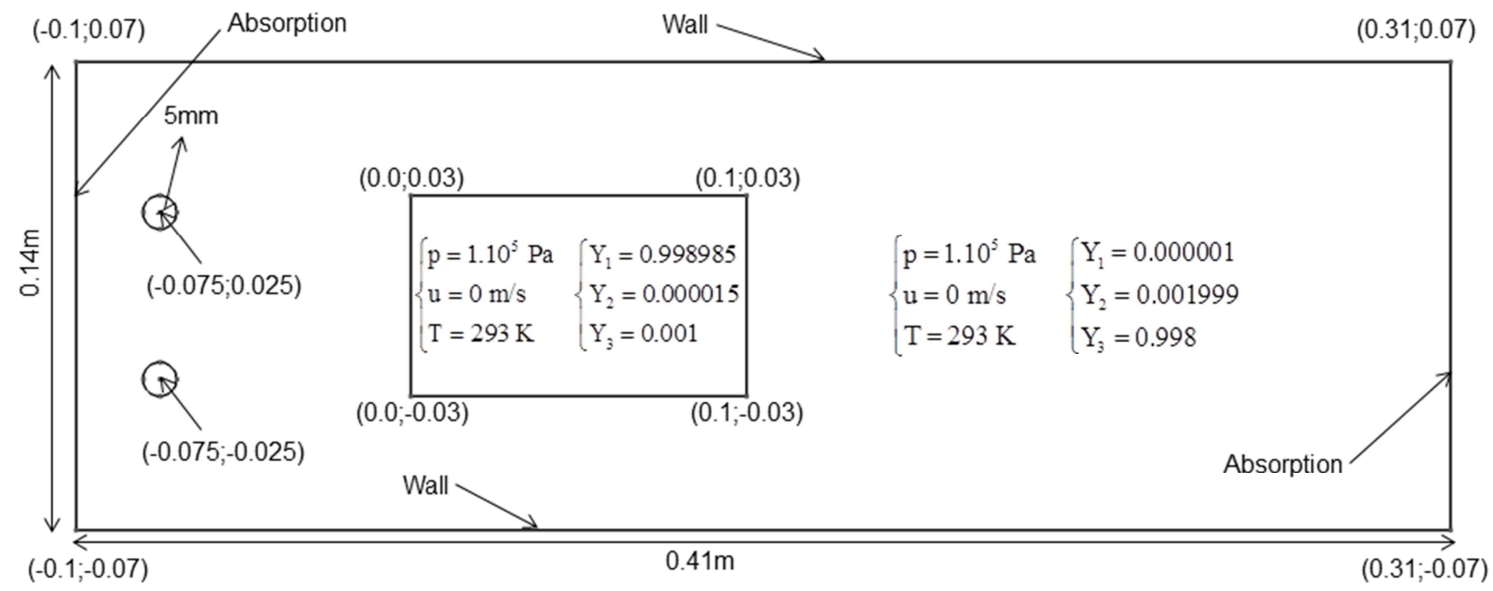

Figure 4.9- Projectiles impact at high velocity onto a water tank in the air. Geometrical data and initial and boundary conditions. The upper projectile has initial velocity components $(400,-10)$ while the lower one has $(400,+10)$ in $\mathrm{m} / \mathrm{s}$ units.

Corresponding computational results are shown in Fig. 4.10 at several times.
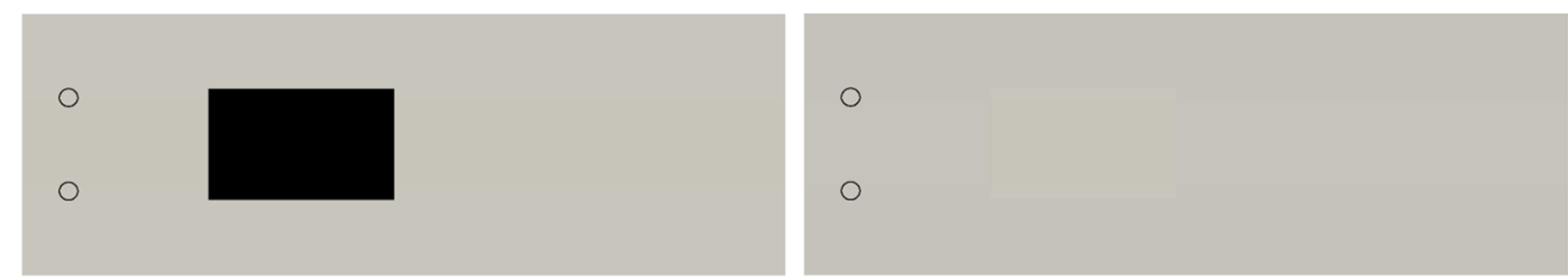

(a)
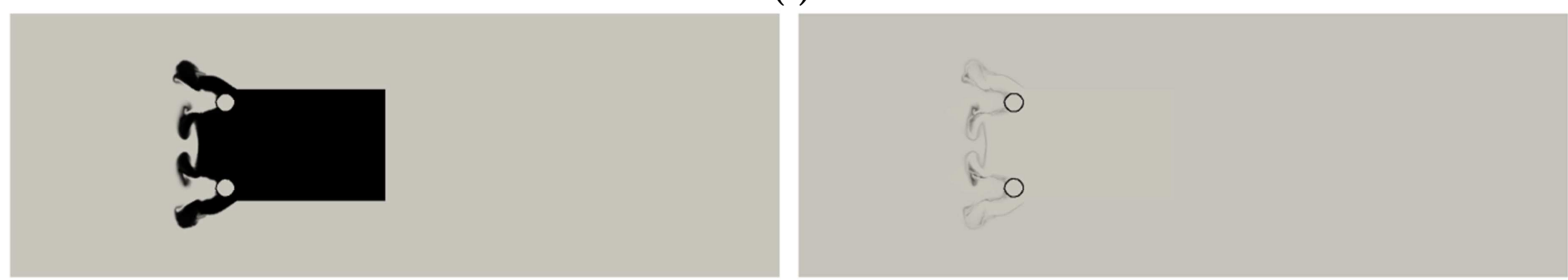

(b)
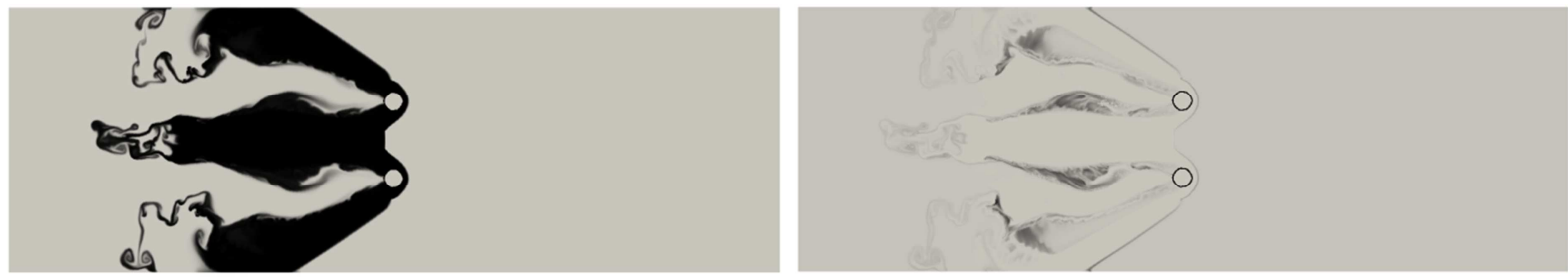

(c) 

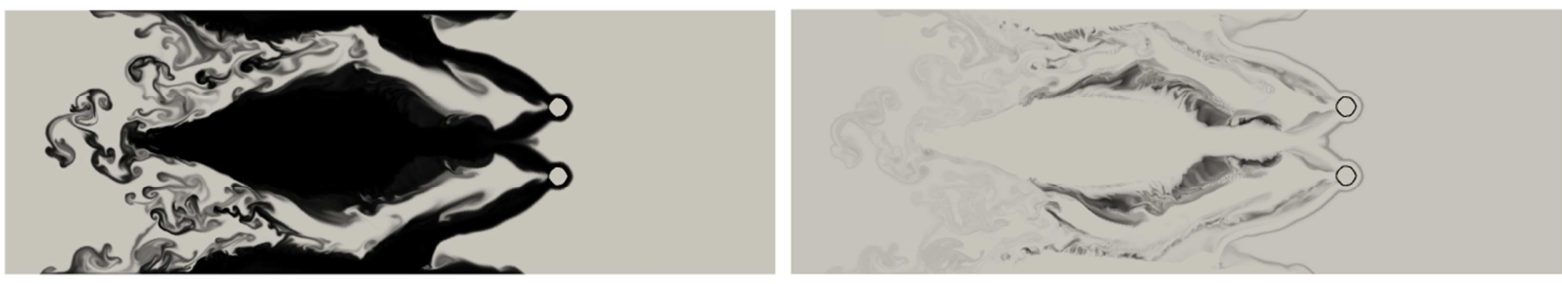

(d)
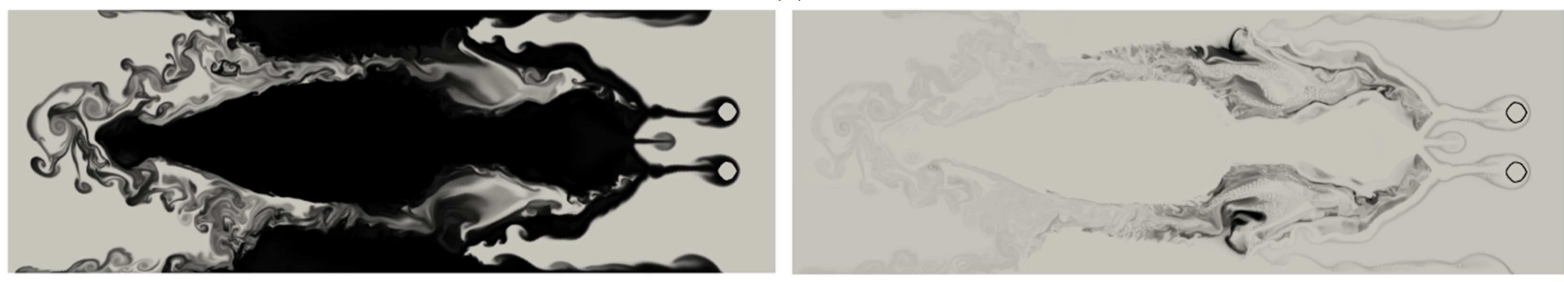

(e)
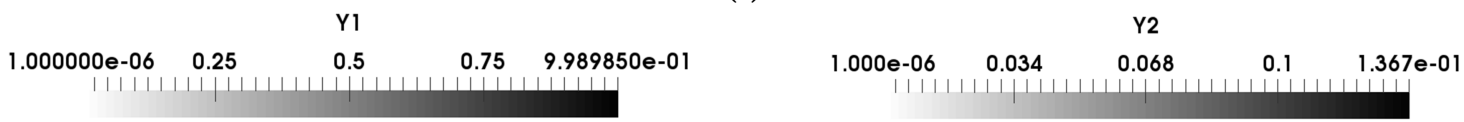

Figure 4.10- Interaction of two projectiles at high speed on a water tank settled in the air. Computed mass fractions of liquid water (left column) and water vapor (right column) appearing during cavitation. Computational parameters: MUSCL scheme with

Overbee limiter for the Level-Set functions, Minmod limiter for the other variables, CFL $=0.5$, number of cells (triangles) $=$ 1489 476. Results are shown at times (a) $0 \mathrm{~ms}$; (b) $0.225 \mathrm{~ms}$; (c) $0.45 \mathrm{~ms}$; (d) $0.675 \mathrm{~ms}$; (e) $0.9 \mathrm{~ms}$.

Fig. 4.10 illustrates method's capabilities where two-phase effects with phase transition are present, in the presence of liquid gas interfaces and solid-fluid interfaces. Extra extension is now addressed with two-way coupling.

\section{5 - Two-way coupling}

The motion of rigid bodies is now considered as coupled to the fluid flow through the pressure force integral over the body surface. The pressure force exerted by the fluid on the solid surface is defined as,

$\overrightarrow{\mathrm{F}_{\mathrm{p}}}=\int_{\partial \Omega_{\mathrm{S}}} \mathrm{p} \overrightarrow{\mathrm{n}} \mathrm{d} S=\mathrm{F}_{\mathrm{px}} \overrightarrow{\mathrm{e}_{\mathrm{x}}}+\mathrm{F}_{\mathrm{py}} \overrightarrow{\mathrm{e}_{\mathrm{y}}}$

where $\partial \Omega_{S}$ denotes the surface of the rigid body.

The cell faces belonging to the rigid boundary surface are detected as:

- For a given face ' $\mathrm{f}$ ' belonging to the entire set of faces of the overall mesh. This face belongs to two neighboring cells, say for instance cells $i$ and $j$.

- If the product of the Level-Set functions $\Phi_{\mathrm{i}} \cdot \Phi_{\mathrm{j}}<0$, then the face belongs to the set of faces of $\partial \Omega_{\mathrm{S}}$.

Consequently the discrete analogue of Eq. (5.1) becomes,

$\overrightarrow{\mathrm{F}}_{\mathrm{p}}=\sum_{\mathrm{f} \in \partial \Omega_{\mathrm{S}}} \mathrm{p}_{\mathrm{f}} \overrightarrow{\mathrm{n}_{\mathrm{f}}} \mathrm{S}_{\mathrm{f}}$

With the help of pressure force exerted on the rigid body its velocity is updated thanks to the Newton's law:

$\overrightarrow{\mathrm{u}_{\mathrm{s}}^{\mathrm{n}+1}}=\overrightarrow{\mathrm{u}_{\mathrm{s}}^{\mathrm{n}}}+\Delta \mathrm{t} \frac{\overrightarrow{\mathrm{F}_{\mathrm{p}}}}{\mathrm{M}}$

As the rigid body velocity is time dependent but independent of space, the Level-Set function still obeys the conservation law, Eq. (2.3). The overall algorithm described in Sections 3 and 4 is thus unchanged.

The method is now illustrated on various flow configurations. An array of 6 cylindrical particles of radius $r$ $=5 \mathrm{~mm}$ is considered and set to motion under shock wave interaction. The mass of each particle is arbitrarily set to $\mathrm{M}=0.8 \mathrm{~g}$ and are initially settled in air at atmospheric conditions. Each particle is tracked 
by its own Level-Set function, different for each particle. At the left boundary of the domain, piston conditions are adopted corresponding to a shock wave emitted to the gas at Mach number 1.24. The various initial and boundary conditions are given in Fig. 5.1. In the first run, the particles are aligned.

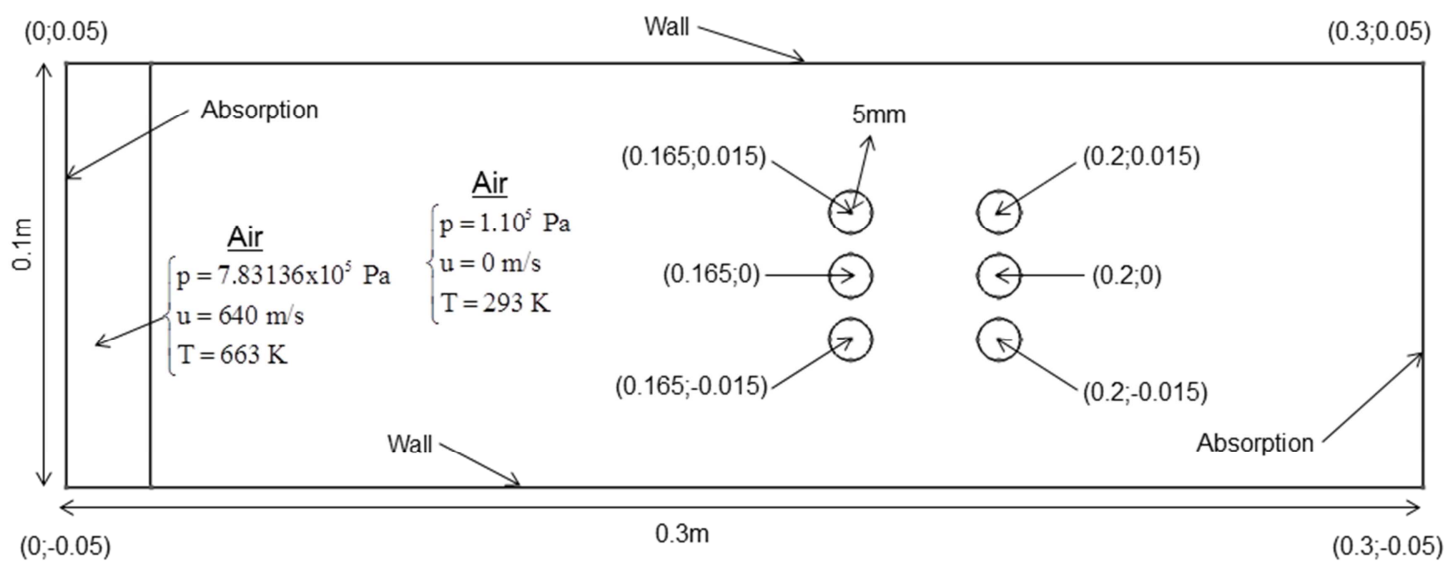

Figure 5.1- Two-way coupling illustration 1 - Shock interaction with an array or aligned particles. Initial data and boundary conditions.

As before, the MUSCL scheme is used with Overbee limiter for the Level-Set function and Minmod for the other flow variables. The pressure contours resulting of the shock interaction are shown at various times in the Figure 5.2.

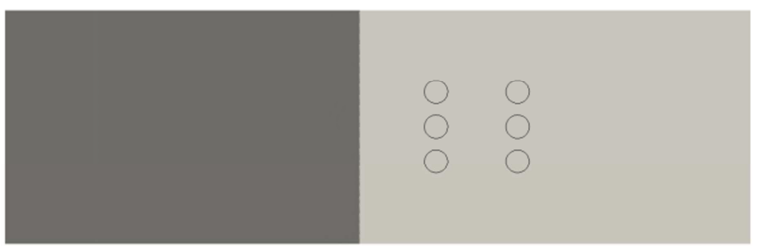

(a)

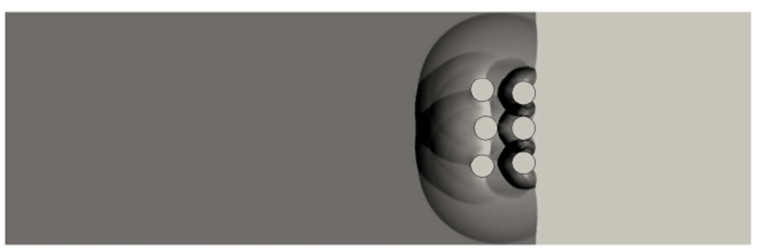

(c)

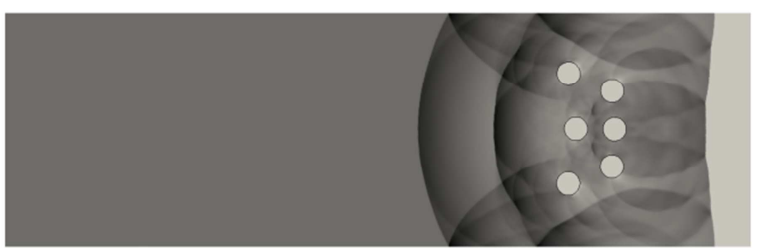

(e)

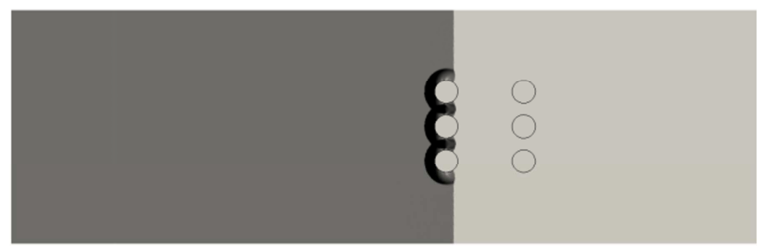

(b)

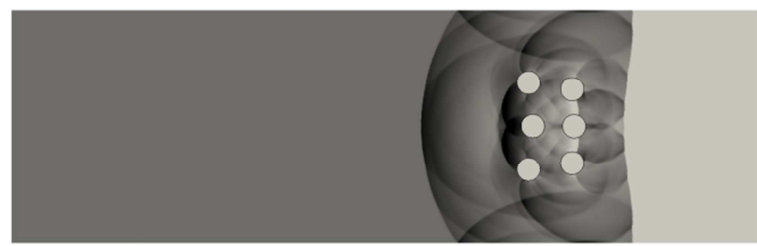

(d)

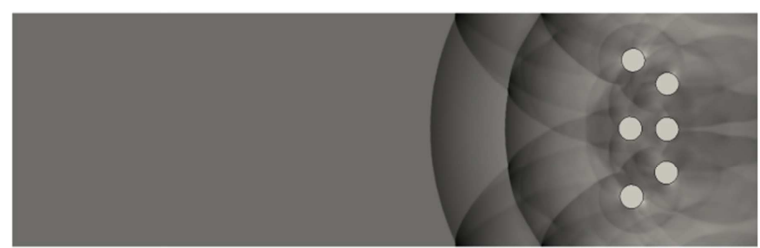

(f)

\section{$\mathrm{P}(\mathrm{Pa})$}

$\begin{array}{lllll}4.039 e+05 & 6.13 e+5 & 8.23 e+5 & 1.03 e+6 & 1.242 e+06\end{array}$

$|1| 1|1| 1|||||||||||+||+|+|+|+|+|+|+|+|+|$

Figure 5.2- Pressure contours resulting of the shock interaction with an array of aligned rigid solid particles. The mesh involves 615278 cells corresponding to an average space size of $0.2 \mathrm{~mm}$. Both transmitted and reflected shock waves are clearly visible as well as the motion of the various particles, no longer aligned during time evolution. The results are shown at times (a) $0.147 \mathrm{~ms}$; (b) $0.189 \mathrm{~ms}$; (c) $0.231 \mathrm{~ms}$; (d) $0.273 \mathrm{~ms}$; (e) $0.315 \mathrm{~ms}$; (f) $0.357 \mathrm{~ms}$.

At each interaction with a particles layer a reflected shock is emitted. Indeed, after the passage of the first particles layer, the shock reforms very quickly and interacts with the second layer, resulting in both 
transmitted and reflected shock waves. The transmitted shock reforms quickly to a discontinuous wave, while the reflected one stays a train of shock waves during the physical time observed.

It is interesting to note the very good symmetry of the computations while achieved on unstructured grids, this observation giving confidence to the coupling method.

The same type of initial configuration with staggered particles is considered for a second run, as shown in Fig. 5.3.

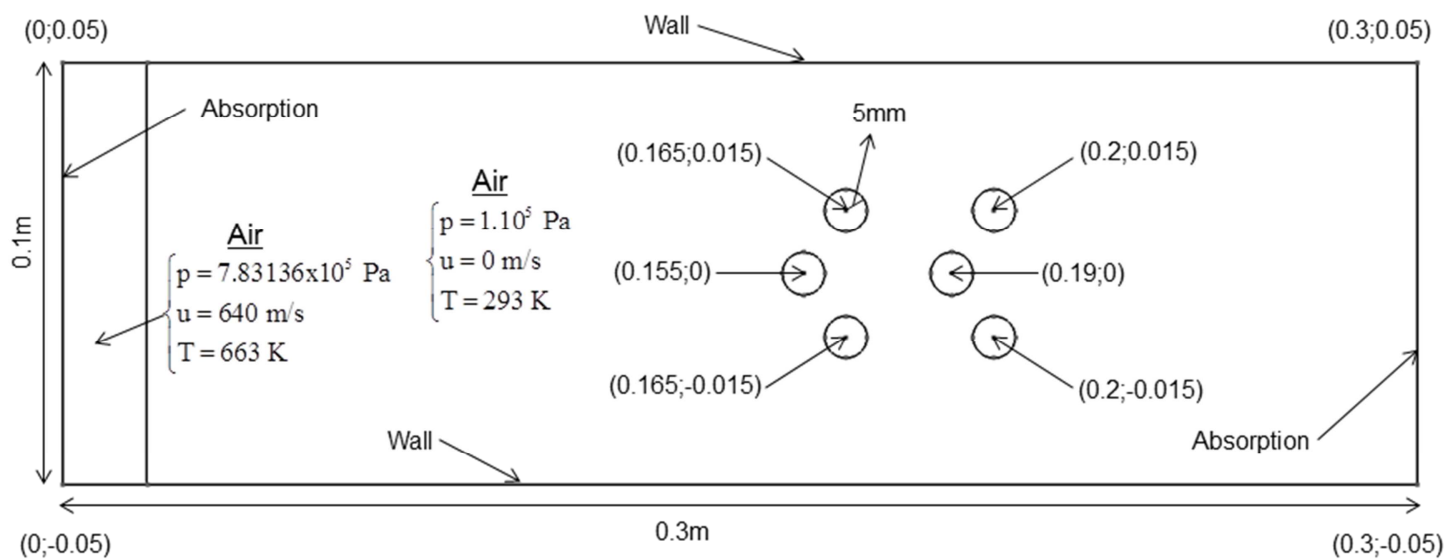

Figure 5.3- Two-way coupling illustration 2 - Shock interaction with an array or staggered particles. Initial data and boundary conditions.

Associated computational results are shown in Fig. 5.4. About the same mesh and grid spacing as before are used as well as computational parameters of the MUSCL and coupling methods.

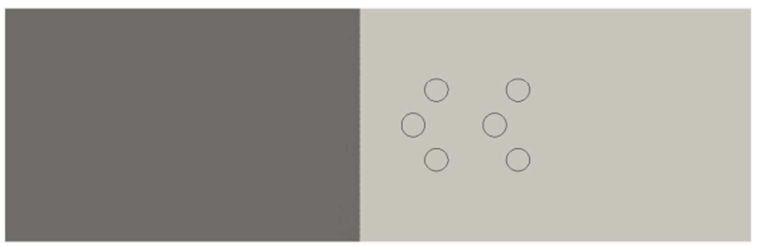

(a)

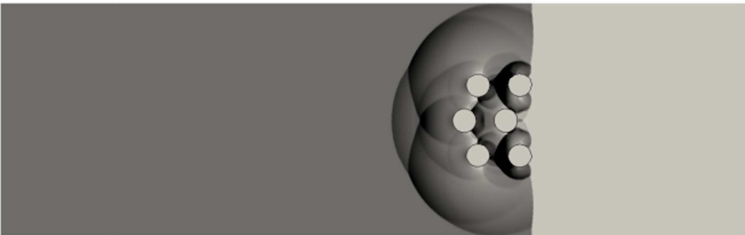

(c)

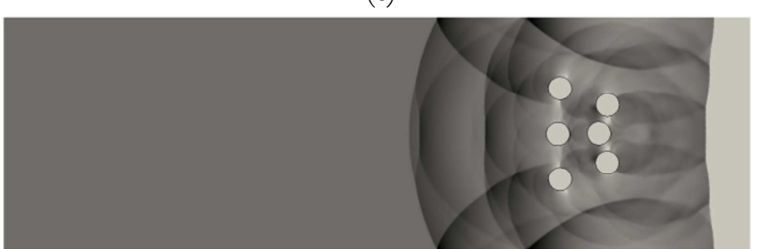

(e)

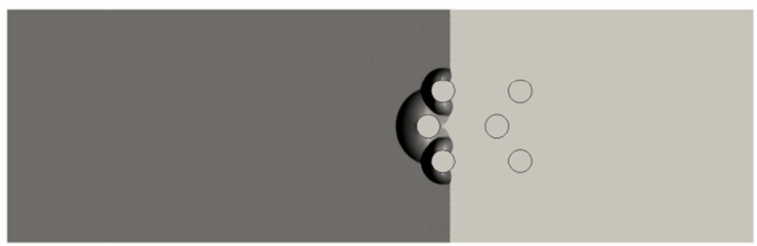

(b)

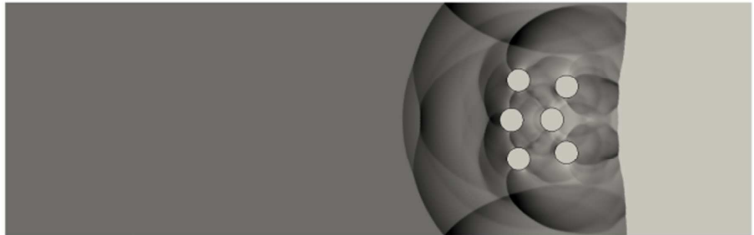

(d)

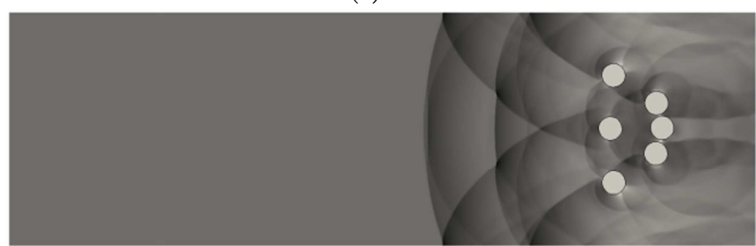

(f)

\section{$\mathrm{P}(\mathrm{Pa})$}

$\begin{array}{lllll}4.039 e+05 & 6.13 e+5 & 8.23 e+5 & 1.03 e+6 & 1.242 e+06\end{array}$

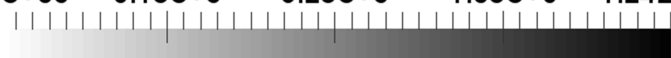

Figure 5.4- Pressure contours resulting of the shock interaction with an array of staggered rigid solid particles. The mesh involves 616276 cells corresponding to an average space size of $0.2 \mathrm{~mm}$. Both transmitted and reflected shock waves are clearly visible as well as the motion of the various particles that tend to form a cluster. The results are shown at times (a) $0.147 \mathrm{~ms}$; (b) $0.189 \mathrm{~ms}$; (c) $0.231 \mathrm{~ms}$; (d) $0.273 \mathrm{~ms}$; (e) $0.315 \mathrm{~ms}$; (f) $0.357 \mathrm{~ms}$. 
The same observations as for the previous test are valid. An extra interesting feature appears with the particle layer at right that tends to form a cluster due to the interactions with the fluid flow.

\section{Conclusion}

A Level-Set type method has been developed to track rigid bodies on unstructured meshes. Thanks to the Overbee limiter of Chiapolino et al. (2017) the method doesn't need reinitialization, nor interface reconstruction. A solid fluid coupling method has been built and compared to other approaches, based on stiff relaxation and conventional Ghost-Cell extrapolation. It is simple to implement and improves convergence. It has been extended to 2D and validated against 2D computations of supersonic two-phase flow around blunt body at rest. The overall method has been extended to two-way coupling and illustrations have been shown.

Acknowledgements. Part of this work has been supported by Excellence Initiative of Aix-Marseille University - A*MIDEX, a French "Investissements d'Avenir" programme, in the framework of the Labex MEC.

\section{References}

Abgrall, R., Beaugendre, H., \& Dobrzynski, C. (2014). An immersed boundary method using unstructured anisotropic mesh adaptation combined with level-sets and penalization techniques. Journal of Computational Physics, 257, 83-101.

Barth, T., \& Jespersen, D. (1989) The design and application of upwind schemes on unstructured meshes. In 27th Aerospace sciences meeting (p. 366)

Baum, J., Luo, H., \& Loehner, R. (1994). A new ALE adaptive unstructured methodology for the simulation of moving bodies. In 32nd Aerospace Sciences Meeting and Exhibit (p. 414).

Chiapolino A., Boivin P., \& Saurel R. (2017) A simple and fast phase transition relaxation solver for compressible multicomponent two-phase flows, Computers and Fluids, 150, 31-45

Chiapolino A., Boivin P., \& Saurel R. (2017) A simple phase transition relaxation solver for liquidvapor flows, International Journal for Numerical Methods in Fluids, 83(7), 583-605

Chiapolino, A., Saurel, R., Nkonga, B. (2017) Sharpening diffuse interfaces with compressible fluids on unstructured meshes. Journal of Computational Physics, 340, 389-417

Chinnayya, A., Daniel, E., \& Saurel, R. (2004) Modelling detonation waves in heterogeneous energetic materials. Journal of Computational Physics, 196(2), 490-538

Farhat, C., Rallu, A., \& Shankaran, S. (2008) A higher-order generalized ghost fluid method for the poor for the three-dimensional two-phase flow computation of underwater implosions. Journal of Computational Physics, 227(16), 7674-7700

Farhat, C., Gerbeau, J. F., \& Rallu, A. (2012) FIVER: A finite volume method based on exact two-phase Riemann problems and sparse grids for multi-material flows with large density jumps. Journal of Computational Physics, 231(19), 6360-6379.

Fedkiw, R. P., Aslam, T., Merriman, B., Osher, S. (1999) A non-oscillatory Eulerian approach to interfaces in multimaterial flows (the Ghost Fluid Method). Journal of Computational Physics, 152(2), 457-492

Glimm J, Grove J, Li X, Shyue K, Zeng Y, Zhang Q. (1998) Three-dimensional front tracking. SIAM Journal of Scientific Computing. 19:703-27

Le Martelot, S., Saurel, R., \& Nkonga, B. (2014) Towards the direct numerical simulation of nucleate boiling flows. International Journal of Multiphase Flow, 66, 62-78

Le Métayer, O., Massoni, J., \& Saurel, R. (2004) Élaboration des lois d'état d'un liquide et de sa vapeur pour les modèles d'écoulements diphasiques. International journal of thermal sciences, 43(3), 265-276

Le Métayer, O., and Saurel, R. (2016) The Noble-Abel stiffened-gas equation of state. Physics of Fluids, 28(4), 046102

Liu, T. G., Khoo, B. C., \& Yeo, K. S. (2003) Ghost fluid method for strong shock impacting on material 
interface. Journal of Computational Physics, 190(2), 651-681

Liu, T. G., Khoo, B. C., \& Xie, W. F. (2006) The modified ghost fluid method as applied to extreme fluidstructure interaction in the presence of cavitation. Communications in Computational Physics, 1(5), 898-919

Liu, T. G., Ho, J. Y., Khoo, B. C., \& Chowdhury, A. W. (2008) Numerical simulation of fluid-structure interaction using modified ghost fluid method and Naviers equations. Journal of Scientific Computing, 36(1), 45-68

Nkonga, B., \& Guillard, H. (1994) Godunov type method on non-structured meshes for threedimensional moving boundary problems. Computer methods in applied mechanics and engineering, 113(1-2), 183-204

Nkonga, B. (2000) On the conservative and accurate CFD approximations for moving meshes and moving boundaries. Computer methods in applied mechanics and engineering, 190(13-14), 1801-1825

Olsson E, Kreiss G, Zahedi S. (2007) A conservative level set method for two phase flow II. Journal of Computational Physics. 225:785-807

Saurel, R., Boivin, P., \& Le Métayer, O. (2016) A general formulation for cavitating, boiling and evaporating flows. Computers \& Fluids, 128, 53-64

Shukla RK, Pantano C, Freund JB. (2010) An interface capturing method for the simulation of multi-phase compressible flows. J. Comput. Phys. 229:7411-39

Sweby, P. K. (1984) High resolution schemes using flux limiters for hyperbolic conservation laws. SIAM Journal on Numerical Analysis, 21(5), 995-1011

Toro, E. F., Spruce, M., \& Speares, W. (1994) Restoration of the contact surface in the HLLRiemann solver. Shock waves, 4(1), 25-34.

Van Leer, B. (1979) Towards the ultimate conservative difference scheme. V. A second-order sequel to Godunov's method. Journal of Computational Physics, 32(1), 101-136

Wang, C. W., Liu, T. G., \& Khoo, B. C. (2006) A real ghost fluid method for the simulation of multimedium compressible flow. SIAM Journal on Scientific Computing, 28(1), 278-302

Wang, K., Rallu, A., Gerbeau, J. F., \& Farhat, C. (2011) Algorithms for interface treatment and load computation in embedded boundary methods for fluid and fluid-structure interaction problems. International Journal for Numerical Methods in Fluids, 67(9), 1175-1206

Wood, A. B. (1930) A Textbook of Sound. G. Bell and Sons Ltd., London

Youngs, D. L. (1984) An interface tracking method for a 3D Eulerian hydrodynamics code. Atomic Weapons Research Establishment (AWRE) Technical Report, (44/92), 35

Zalesak, S. T. (1979) Fully multidimensional flux-corrected transport algorithms for fluids. Journal of Computational Physics, 31(3), 335-362

Zeng, X., \& Farhat, C. (2012) A systematic approach for constructing higher-order immersed boundary and ghost fluid methods for fluid-structure interaction problems. Journal of Computational Physics, 231(7), 2892-2923 